\title{
Active Involvement of People with Dementia: A Systematic Review of Studies Developing Supportive Technologies
}

\author{
Sandra Suijkerbuijk ${ }^{\mathrm{a}, \mathrm{b}, *}$, Henk Herman Nap ${ }^{\mathrm{a}, \mathrm{b}}$, Lotte Cornelisse ${ }^{\mathrm{a}}$, Wijnand A. IJsselsteijn ${ }^{\mathrm{b}}$, \\ Yvonne A.W. de Kort ${ }^{\mathrm{b}}$ and Mirella M.N. Minkman ${ }^{\mathrm{a}, \mathrm{c}}$ \\ ${ }^{a}$ Vilans, Centre of expertise Long-term care, Utrecht, The Netherlands \\ ${ }^{\mathrm{b}}$ Human Technology Interaction, University of Technology, Eindhoven, The Netherlands \\ ${ }^{\mathrm{c}}$ Tilburg University, TIAS School for Business and Society, Tilburg, The Netherlands
}

Handling Associate Editor: Francesca Baglio

Accepted 8 April 2019

\begin{abstract}
Although there are promising benefits of supportive technology in dementia care, use of these technologies is still limited. It is challenging for researchers and developers in this field to actively involve people with dementia in development. This review updates and builds on existing knowledge by including a contemporary and relevant perspective. This perspective was gained by including search words and search databases from the field of Human Computer Interaction (HCI) and Design, as these fields were expected to supply novel insights in the complex task of actively involving people with dementia in developing supportive technologies. A total of 49 out of 3456 studies were included which describe the development of a great variety of technologies. Often people with dementia were involved in the generative or evaluative phase of the development. Interviews and observations were most commonly used methods. In seven articles the people with dementia were co-designers. This literature review reflects that people with dementia can influence the development of technology in regards to content, design, and even the initial idea, although the impact on how they experience their own involvement remains largely unknown. There is a lack of specific knowledge on appropriate methods and materials for active involvement of people with dementia in supportive technology development, even when including articles from the field of HCI and Design. Future research is needed to further appreciate and improve the desired role of people with dementia in meaningful technology development.
\end{abstract}

Keywords: Co-design, dementia, patient participation, supportive technology

\section{INTRODUCTION}

There are over 50 million people living with dementia worldwide, and this number is still increasing [1]. For people with dementia, everyday life is full of challenges due to difficulties with memory, thinking, orientation, language, comprehension, action, and judgement. Moreover, these impairments

${ }^{*}$ Correspondence to: Sandra Suijkerbuijk, Vilans, Centre of expertise Long-term care, PO Box 82283503 RE Utrecht, The Netherlands. E-mail: s.suijkerbuijk@vilans.nl. become more salient over time. For both the person with dementia, as well as their informal caregivers, the syndrome has a great impact on their experienced well-being [2]. Since there is currently no cure, there is an urgent need for interventions that support independence and well-being in everyday life.

\section{The potential benefits of supportive technology}

Technology is often suggested as a means to accomplish the aforementioned support. The 
development of supportive technologies is seen as a worldwide priority in large national and international funding programs. For instance, the European Active and Assisted Living (AAL) program has a total budget of $€ 700$ million to distribute between 2014 and 2020 [3]. Examples of supportive technologies that are developed in these programs are sensor systems, smartphones with low complexity, reminiscence applications, and electronic calendars. These (or combinations of) technologies can promote safety, foster communication, provide multi-sensory stimulation, or act as memory aids [4]. However, despite their apparent benefits, the use of supportive technologies in dementia care is still limited. Low adoption rates of assistive technology may signal unmet user needs and wishes in product design.

The risk of inappropriate, unappealing, or less usable products is of course higher as a designer's perceptions and competences diverge farther from those of the users' gap which is probably wider when designing for users with dementia. This might result in products with a too strong focus on feelings of safety (e.g., monitoring risk), instead of improving the experience of the impairment itself [5]. Furthermore, for progressive syndromes, such as dementia, current supportive technologies might not be sufficiently flexible to serve the changing needs of their users. Moreover, there is a great variety within the group 'people with dementia', such as differences in demographics (e.g., socioeconomic status) and personality (e.g., acceptance of the diagnosis), but also due to the diversity of specific diseases, each with different behavioral, cognitive, and emotional consequences. In itself, this diversity already creates a great challenge for researchers and developers aiming to design technologies that people with dementia truly want and are able to use. It is, therefore, vital to have extensive insight in the dynamic needs, wishes, and abilities of people with dementia.

\section{The different human-centered design practices}

For the general population, the collection of user insights is typically supported through humancentered design (HCD) practices [6]. A successful design practice is guided by design research [7], which are two distinct but interconnected activities in technology development. Outcomes of the design practices are used as research material (researchthrough-design), and research insights are preferably further applied into design outcomes to steer the development. This iterative process can be divided in several phases, all with their own objectives and types of design research. One example of such an iterative design process is provided by Sanders and Stappers [8] and approaches the role of the user as co-designer. HCD can have different of these approaches, such as participatory design, ethnography, empathic design, and co-design [9]. These approaches differ in the nature and intensity of the relationship between designers and users. For instance, is the user an informant or considered an equal partner and is the focus merely on research (what is) or on design (what could be). Strong HCD promises better products by closing the gap between designers and users. But, core symptoms of the dementia syndrome, e.g., lowered capacity in retrospective and abstract thinking, present a major challenge for the different approaches of human-centered design practices with people with dementia [10].

\section{Involving people with dementia in development of technology}

Earlier literature reviews show the importance of involving people with dementia and their informal caregivers in the development of new supportive or assistive technologies [11-14]. They also suggest that, in the majority of studies, people with dementia have had a rather passive role in the development phase, at best serving as an object of study or informant. Their involvement generally occurs in the evaluation phases of technology development and renders insights in the effectiveness, usefulness and acceptability of the developed devices. Few studies report on the involvement of people with dementia throughout the entire development process, e.g., as a continuous equal partner or co-designer. The lack of this involvement is noteworthy since co-design practiced from the start of the development process can have an impact with positive, long-range consequences on the user experience of the eventual design outcome [15]. Co-design with people with dementia can yield an enhanced sense of control in participants [16] and can ultimately lead to a more empathic understanding of the user group [17]. Empathic understanding enables designers and developers to gain fully relevant and intimate user insights needed for more meaningful and suitable technology development. However, involving people with dementia as co-designers in the development can be challenging due to the characteristics of the syndrome, which vary over time and between different diseases implied under the umbrella term 'demen- 
tia'. Being a co-designer might require certain levels of sensory, cognitive, and motoric abilities, which decrease for a person with dementia [18]. The large variety within and between people with dementia adds to the overall challenge of successful co-design with this user group.

\section{A broader perspective needed for state-of-the-art knowledge}

There is limited knowledge about the specific research methods and materials needed to actively involve people with dementia in the entire development process of meaningful supportive technologies. Either successful co-design may be non-existent, or alternatively, the latest studies on successful co-design with people with dementia are not systematically reviewed yet. The most recent systematic review on methodology in this field was performed before 2011 [12]. Because of the increased interest in developing supportive technologies in recent years, we expect that a substantial amount of new research has been performed. Moreover, the commonly used search engines (Cochrane library, PubMed, EMBASE, and CINAHL database) may have limited the outcomes to the field of medical and healthcare research and, therefore, potentially lack relevant contributions from the areas of Human Computer Interaction (HCI) and Design (such as the proceedings of CHI Human Factors in Computing Systems conference). Since the development of supportive technology is an inherently interdisciplinary process, it is expected that relevant complementary knowledge will be available when including research from HCI and Design fields. In addition, designers are well appreciated in their ability to embrace complexity [15], which might give useful insights in how to involve people with dementia in developing meaningful supportive technologies.

The aim of our review is therefore to update and expand the existing body of knowledge by including a new and relevant perspective. This perspective represents an additional step toward achieving a more complete understanding of how people with dementia can have an active role in the different phases of development of new supportive technologies. We provide a detailed analysis on how the different fields use research methods and materials to support people with dementia in their role as an active informant or co-designer in the design and development of supportive technologies. The impact of these roles and research methods on the developed technology is presented as well as the impact on the experiences of people with dementia in the different development phases. To complement the earlier reviews, the following questions are addressed:

1. In which phase of development of technology are people with dementia actively involved?

2. What specific role do people with dementia have in the development?

3. Which research methods and materials are used to involve people with dementia?

4. What is the impact of the active involvement on the technology development and on the participants themselves?

\section{MATERIALS AND METHODS}

\section{Search strategy}

Our initial search strategy was based on the search strategy of the most recent review on co-design with people with dementia of Span and colleagues [12]. They created search strings within three categories: 'dementia', 'technology', and 'involvement' and used the search engines Cochrane library, PubMed, PsycInfo, EMBASE, and CINAHL. For the purpose of the current review, we extended this existing search strategy on three levels by: 1) the time-frame, 2) the search keywords, and 3) the search engines. First, our extended timeframe also includes articles published from July 2011 to July 2017. Second, the search strings were extended in the categories 'technology' (by adding new words, such as, 'platforms', 'sensors', 'robotics') and 'involvement' (e.g., 'co-design', 'service design', 'brainstorm'). Third, the search was also performed in other databases (ACM Digital Library and Web of Science) to expand the review to the field of HCI and design. To gather insight in the value of the added search strings and added databases, we performed separate searches in the time-frame of the review of Span and colleagues [12] as well (including papers until July 2011). An overview of the different searches is shown in Table 1. The searches were first performed in PubMed. Strings from Pubmed were later translated for use in the other databases. The Supplementary Material shows the complete (new) search string.

\section{Selection of studies}

We selected articles written in English. Articles were only included when 1) they described (parts of) 
Table 1

The current review adds to existing work in terms of timeframe (search 1), search keywords (search 2), and search engines (search 3 ) and resulted in 49 new articles

\begin{tabular}{|c|c|c|c|c|}
\hline \multirow{2}{*}{$\begin{array}{l}\text { Search engines } \\
\text { Search } \\
\text { keywords }\end{array}$} & \multicolumn{2}{|c|}{ Cochrane library, PubMed, PsycInfo, EMBASE, \& CINAHL } & \multicolumn{2}{|c|}{ ACM Digital library \& Web of Science } \\
\hline & $\begin{array}{l}\text { 'dementia', 'technology', and } \\
\text { 'involvement' }\end{array}$ & + new search keywords & $\begin{array}{l}\text { 'dementia', 'technology', } \\
\text { and 'involvement' }\end{array}$ & + new search keywords \\
\hline $\begin{array}{l}\text { From inception } \\
\text { to July } 2011\end{array}$ & $\begin{array}{l}\text { Total: } 1039 \\
\text { Without duplicates: } 893 \\
\text { Included: } 26 \\
{[12]}\end{array}$ & $\begin{array}{l}\text { Total: } 1362 \\
\text { Total of new articles: } 279 \\
\text { Included: } \mathbf{2}\end{array}$ & $\begin{array}{l}\text { Total: } 595 \\
\text { Total of new articles: } 406 \\
\text { Included: } 6\end{array}$ & $\begin{array}{l}\text { Total: } 956 \\
\text { Total of new articles: } 258 \\
\text { Included: } \mathbf{3}\end{array}$ \\
\hline \multirow[t]{2}{*}{$\begin{array}{l}\text { From July } 2011 \\
\text { - July } 2017\end{array}$} & $\begin{array}{l}\text { Total: } 1484 \\
\text { Total of new articles: } 1193 \\
\text { Included: } \mathbf{2 1}\end{array}$ & $\begin{array}{l}\text { Total: } 1868 \\
\text { Total of new articles: } 382 \\
\text { Included: } \mathbf{1}\end{array}$ & $\begin{array}{l}\text { Total: } 798 \\
\text { Total of new articles: } 552 \\
\text { Included: } \mathbf{1 3}\end{array}$ & $\begin{array}{l}\text { Total: } 1295 \\
\text { Total of new articles: } 385 \\
\text { Included: } \mathbf{3}\end{array}$ \\
\hline & Search 1 & Search 2 & \multicolumn{2}{|c|}{ Search 3} \\
\hline
\end{tabular}

a development process for new supportive technology meant to be used by people with dementia, and when 2) people with dementia were actively involved in this process (either as active informant or co-designer). We excluded literature reviews and other overview papers describing reflections on projects without actual application of outcomes in the development of technology. Titles and abstracts were independently screened (by the first and third author) to identify relevant articles. Full texts were obtained to check when abstracts missed required information to decide for inclusion or exclusion. Eventually, full-text articles were assessed for eligibility by two reviewers (the first and second author) by using a relevance score on a scale of 0 (not relevant at all) to 10 (very relevant). This relevance score identified whether new and clearly reported outcomes qualified for further analysis. Studies with a relevance mean score below 5 were excluded. When the difference between relevance ratings of both researchers transcended the limits of agreement (mean $\pm 1.96 * S D$ ) [19], the discrepancy was resolved through discussions until consensus was reached.

\section{Quality assessment}

The included articles were evaluated on content and methodology. To appraise the methodological quality of the articles, we used the Mixed Methods Appraisal Tool (MMAT) from [20]. This tool is suitable for all study designs and was, therefore, appropriate for the current review. The first author rated the methodological quality criteria individually. These were discussed with the second author. Scores (from 0 to 100) and a descriptive summary of the use of the MMAT criteria can be found in the results section.

\section{Analysis}

The following information was gathered from the selected articles: name of the overall project, the type of technology developed, the characteristics of participants with dementia, the method of study and the quality score. It was checked whether an informal caregiver was involved, since they can play an important role in the participation of people with dementia. To answer the research questions of this review, an inventory was made of different elements including the phases of technology development, the role of the person with dementia, the used research methods and materials and the subsequent impact of this role on the technology and the person themselves.

\section{Phases of technology development}

To assess in which phase of the development the person with dementia had a role, the design research process described by Sanders and Stappers [8] was used. This design process starts with a pre-design phase (phase 1) that aims at understanding people's experiences in the context of their lives. In the generative phase (phase 2), the first ideas of a new design are constructed by performing co-design activities. In the evaluative phase (phase 3), the iterative development of the technology occurs and users test different prototypes. The actual use of the new technology is researched in the post-design phase (phase 4). 


\section{Role of people with dementia}

We assessed the level of involvement of people with dementia in the development of supportive technology. In accordance with earlier work [12], the role of the person with dementia was identified either as an active informant or as a co-designer [21]. When a user is an active informant, the researcher uses methods such as interviews to generate knowledge to use in the development process. When a user is a codesigner, the user is an equal partner in the process and plays a crucial role multiple phases of the process to develop knowledge, generate ideas and co-develop concepts.

\section{Research methods and materials in the phases of development}

The research methods used in the articles were gathered and indexed. If reported, the type of the method was also identified (e.g., structured or semistructured interviews). Besides the research methods, the materials used in applying these methods were included in the overview (e.g., elicitation material). In case prototypes of the technology were used, these were categorized based on their level of functioning. We discriminate between low-fidelity prototypes (mock-ups, Wizard-of-Oz prototypes) and highfidelity prototypes (alpha prototypes, beta prototypes, and fully functional prototypes) corresponding to the work of Preece and colleagues [22]. Low-fidelity prototypes are simple, cheap, and quick to produce and easily modified. These types of prototypes can be built by paper and pencil and are used in the earlier phases of development in order to get feedback from users on early ideas and concepts. The Wizard-of-Oz prototype is a system operated by a man-behind-thescenes, appearing to be fully functional. On the other hand, high-fidelity prototypes actually use the platforms and technology of the eventual product. These prototypes are therefore also good for testing the technical functioning.

\section{Impact of involvement on technology and participants}

Next, the impact of the involvement of people with dementia on the technology that was developed was defined as one of three categories: no directly reported impact (0), one aspect was changed (1), or multiple aspects were changed (2+). This impact includes, for example, the user requirements or interface improvements derived from the research activities. Next to the outcomes for the supportive technology, the involvement of people with dementia was expected to have an impact on themselves. Whenever described in the article, their experiences were categorized as negative $(-)$ or positive $(+)$.

\section{RESULTS}

The search in the digital libraries was conducted in July 2017. Figure 1 presents the flow chart for the selection of articles. In total, 49 articles were included in the final review. Among the 49 articles, 40 describe a qualitative research study (24 qualitative description, 10 case studies, three ethnography, one narrative and three combined different types of studies). Eight articles describe a mixed method study (six embedded design, one sequential design and one case report). Furthermore, also one quantitative descriptive study was included. The MMAT scores varied among the included studies: 13 articles scored 100\%, 12 articles scored 75\%, 10 articles scored 50\%, 13 articles scored $25 \%$, and one study scored $0 \%$. These latter scores were mainly given to studies with unclear research questions and/or ill-reported data analysis. In Table 2 the characteristics of the articles are summarized.

The articles differed by the purpose of supportive technologies (as can be seen in Table 3), the number of people with dementia included in the study, and the stage of dementia of these participants.

In ten out of 49 articles the numbers of participants with dementia were not or only partly reported, mainly when multiple (observational) sessions in intramural care settings were performed [23-32]. Several articles (including [29, 33]) report reasons for a relatively low number of participants in their study, which can be categorized in issues related to the health status of the person with dementia, difficulties with technology or the planning of the study. The level of dementia of the participants is usually defined in stages. From the 49 articles, 16 articles involved people in mild to moderate stages. Other reported stages were mild $(n=7)$, mild to severe $(n=5)$, moderate to severe $(n=2)$, and moderate $(n=1)$. When the stage of dementia is reported $(n=31)$, only 22 studies report some form of clinical measurements; the Mini-Mental State Examination (MMSE) [34] score was used most often $(n=13)$. A large number of studies $(n=18)$ did not report the stage of dementia at all. In 37 of the 49 articles the informal caregiver was also actively involved as a participant, for example, by being interviewed or as support in the research activity with the person with dementia. 
Table 2

Description of included articles

\begin{tabular}{|c|c|c|c|c|c|c|c|c|}
\hline \multirow[t]{2}{*}{ Search } & \multirow{2}{*}{$\begin{array}{l}\text { Publication Author } \\
\text { Project [Ref] }\end{array}$} & \multirow{2}{*}{$\begin{array}{l}\text { Technology } \\
\text { developed }\end{array}$} & \multicolumn{3}{|c|}{ Main characteristics of participants with dementia } & \multirow{2}{*}{$\begin{array}{l}\text { Informal } \\
\text { caregiver as } \\
\text { participant? }\end{array}$} & \multirow{2}{*}{$\begin{array}{l}\text { Research method } \\
\text { (MMAT) }\end{array}$} & \multirow{2}{*}{$\begin{array}{l}\text { Quality } \\
\text { score } \\
\text { (MMAT) }\end{array}$} \\
\hline & & & $\bar{N}=$ & $\begin{array}{l}\text { Stage of } \\
\text { dementia }\end{array}$ & $\begin{array}{l}\text { Living } \\
\text { conditions }\end{array}$ & & & \\
\hline 1 & $\begin{array}{l}\text { Karlsson et al., } 2011 \\
\text { COGKNOW [70] }\end{array}$ & $\begin{array}{l}\text { Platform with } \\
\text { reminder } \\
\text { system, GPS } \\
\text { and sensors for } \\
\text { surveillance }\end{array}$ & 2 & $\begin{array}{l}\text { Mild to } \\
\text { moderate } \\
\text { (MMSE) }\end{array}$ & At home & Yes & $\begin{array}{l}\text { Qualitative research: Case } \\
\text { study }^{1}\end{array}$ & $100 \%$ \\
\hline 1 & $\begin{array}{l}\text { Span et al. } 2017 \\
\text { DecideGuide [47] }\end{array}$ & Website/portal & $\begin{array}{l}\text { Five research } \\
\text { activities: } \\
23 ; 9 ; 12 ; 3 \\
4\end{array}$ & $\begin{array}{l}\text { Mild to } \\
\text { moderate }\end{array}$ & $\begin{array}{l}\text { Five } \\
\quad \text { participants } \\
\text { living in } \\
\text { nursing } \\
\text { home }\end{array}$ & No & $\begin{array}{l}\text { Qualitative research: Case } \\
\text { study }\end{array}$ & $100 \%$ \\
\hline 1 & $\begin{array}{l}\text { Meiland et al. } 2012 \\
\text { COGKNOW [46] }\end{array}$ & $\begin{array}{l}\text { Platform with } \\
\text { reminder } \\
\text { system, GPS } \\
\text { and sensors for } \\
\text { surveillance }\end{array}$ & $\begin{array}{c}\text { Three research } \\
\text { activities: } \\
\text { 16; 14; } 12\end{array}$ & $\begin{array}{l}\text { Mild to } \\
\text { moderate } \\
\text { (MMSE) }\end{array}$ & At home & Yes & $\begin{array}{l}\text { Mixed method research: } \\
\text { Embedded design }^{2}\end{array}$ & $100 \%$ \\
\hline 1 & Lopes et al. 2016 [41] & Item locator & $\begin{array}{l}\text { Five research } \\
\text { activities: } \\
\quad 11 ; 10 ; 4 ; 5 \\
9\end{array}$ & Not reported & At home & Yes & $\begin{array}{l}\text { Qualitative research: } \\
\text { Qualitative description }\end{array}$ & $75 \%$ \\
\hline 1 & $\begin{array}{l}\text { Boman et al. } 2014 \\
\text { [43] }\end{array}$ & $\begin{array}{l}\text { Video } \\
\quad \text { conferencing }\end{array}$ & 4 & $\begin{array}{l}\text { Mild to } \\
\text { moderate } \\
\text { (time since } \\
\text { diagnosis) }\end{array}$ & At home & Yes & $\begin{array}{l}\text { Qualitative research: Case } \\
\text { study }\end{array}$ & $100 \%$ \\
\hline 1 & Wang et al., 2017 [45] & $\begin{array}{l}\text { Tele-operated } \\
\text { robot }\end{array}$ & 10 & $\begin{array}{l}\text { Mild to severe } \\
\text { (MMSE) }\end{array}$ & At home & Yes & $\begin{array}{l}\text { Qualitative research: } \\
\text { Qualitative description }\end{array}$ & $75 \%$ \\
\hline 1 & $\begin{array}{l}\text { Peeters et al., } 2016 \\
\text { [57] }\end{array}$ & $\begin{array}{l}\text { Music } \\
\quad \text { intervention }\end{array}$ & 5 & Mild to severe & Not reported & Yes & $\begin{array}{l}\text { Qualitative research: Case } \\
\text { study }\end{array}$ & $25 \%$ \\
\hline 1 & $\begin{array}{l}\text { Meiland et al., } 2014 \\
\text { ROSETTA [48] }\end{array}$ & $\begin{array}{l}\text { Sensors for } \\
\text { surveillance }\end{array}$ & $\begin{array}{l}\text { Four research } \\
\text { activities: } 3 \text {; } \\
3 ; 4 ; 4\end{array}$ & Not reported & At home & Yes & $\begin{array}{l}\text { Qualitative research: } \\
\text { Qualitative description }\end{array}$ & $75 \%$ \\
\hline 1 & $\begin{array}{l}\text { Suijkerbuijk et al., } \\
2015 \text { [55] }\end{array}$ & Light armature & 12 & Not reported & At home & Yes & $\begin{array}{l}\text { Qualitative research: } \\
\text { Qualitative description }\end{array}$ & $100 \%$ \\
\hline
\end{tabular}




\begin{tabular}{|c|c|c|c|c|c|c|c|c|}
\hline 1 & $\begin{array}{l}\text { Olsson et al., } 2013 \\
\text { [58] }\end{array}$ & $\begin{array}{l}\text { GPS for } \\
\quad \text { localization }\end{array}$ & 5 & $\begin{array}{l}\text { Mild to } \\
\text { moderate } \\
\text { (MMSE) }\end{array}$ & At home & Yes & $\begin{array}{l}\text { Qualitative research: } \\
\text { Qualitative description }\end{array}$ & $100 \%$ \\
\hline 1 & $\begin{array}{l}\text { Boman et al., } 2014 \\
\text { [53] }\end{array}$ & $\begin{array}{l}\text { Video } \\
\text { conferencing }\end{array}$ & $\begin{array}{l}\text { Two research } \\
\text { activities: } 2 \text {; } \\
4\end{array}$ & $\begin{array}{l}\text { Mild to } \\
\text { moderate } \\
\text { (time since } \\
\text { diagnosis) }\end{array}$ & Not reported & Yes & $\begin{array}{l}\text { Qualitative research: } \\
\text { Qualitative description }\end{array}$ & $100 \%$ \\
\hline 1 & $\begin{array}{l}\text { Kerkhof et al., } 2015 \\
\text { [44] }\end{array}$ & Reminder system & 7 & Not reported & $\begin{array}{l}\text { In nursing } \\
\text { home }\end{array}$ & Yes & $\begin{array}{l}\text { Qualitative research: } \\
\text { Qualitative description }\end{array}$ & $100 \%$ \\
\hline 1 & $\begin{array}{l}\text { Hattink et al., } 2016 \\
\text { ROSETTA [54] }\end{array}$ & $\begin{array}{l}\text { Sensors for } \\
\text { surveillance }\end{array}$ & 42 & $\begin{array}{l}\text { Mild to severe } \\
\text { (MMSE) }\end{array}$ & At home & Yes & $\begin{array}{l}\text { Mixed method research: } \\
\text { Embedded design }\end{array}$ & $75 \%$ \\
\hline 1 & $\begin{array}{l}\text { Pakrasi et al., } 2015 \\
\text { [71] }\end{array}$ & $\begin{array}{l}\text { Video } \\
\quad \text { conferencing }\end{array}$ & 26 & Mild to severe & At home & Yes & $\begin{array}{l}\text { Qualitative research: } \\
\text { Qualitative description }\end{array}$ & $25 \%$ \\
\hline 1 & $\begin{array}{l}\text { Dethlefs et al., } 2017 \\
\text { [52] }\end{array}$ & $\begin{array}{l}\text { Cognitive } \\
\text { stimulation }\end{array}$ & 10 & $\begin{array}{l}\text { Mild to } \\
\text { moderate } \\
\text { (CDR) }\end{array}$ & Not reported & No & $\begin{array}{l}\text { Quantitative research: } \\
\text { Quantitative } \\
\text { description }\end{array}$ & $25 \%$ \\
\hline 1 & $\begin{array}{l}\text { Jacova et al., } 2015 \\
\text { [36] }\end{array}$ & Website / Portal & $\begin{array}{l}\text { Two research } \\
\text { activities: } 5 \text {; } \\
6\end{array}$ & Mild (MMSE) & Not reported & No & $\begin{array}{l}\text { Mixed method research: } \\
\text { Embedded design }\end{array}$ & $75 \%$ \\
\hline 1 & $\begin{array}{l}\text { McCabe \& Innes, } \\
\quad 2013 \text { [72] }\end{array}$ & $\begin{array}{l}\text { GPS for } \\
\quad \text { localization }\end{array}$ & 12 & Not reported & Not reported & Yes & $\begin{array}{l}\text { Qualitative research: } \\
\text { Qualitative description }\end{array}$ & $75 \%$ \\
\hline 1 & Wang et al., 2011 [73] & $\begin{array}{l}\text { Automated } \\
\text { wheelchair }\end{array}$ & 6 & $\begin{array}{l}\text { Mild to } \\
\text { moderate } \\
\text { (MMSE) }\end{array}$ & $\begin{array}{l}\text { In nursing } \\
\text { home }\end{array}$ & No & $\begin{array}{l}\text { Mixed method research: } \\
\text { Embedded design }\end{array}$ & $75 \%$ \\
\hline 1 & $\begin{array}{l}\text { Hattink et al., } 2016 \\
\text { [74] }\end{array}$ & Website / Portal & $\begin{array}{l}\text { Three research } \\
\text { activities: } 4 \text {; } \\
\text { 88; } 6\end{array}$ & Not reported & At home & Yes & $\begin{array}{l}\text { Mixed method research: } \\
\text { Embedded design }\end{array}$ & $50 \%$ \\
\hline 1 & $\begin{array}{l}\text { Wolters et al., } 2016 \\
\text { [65] }\end{array}$ & Reminder system & $\begin{array}{l}\text { Two research } \\
\text { activities: } 2 \text {; } \\
4\end{array}$ & Not reported & At home & Yes & $\begin{array}{l}\text { Qualitative research: } \\
\text { Qualitative description }\end{array}$ & $75 \%$ \\
\hline 1 & $\begin{array}{l}\text { Begum et al., } 2013 \\
\text { Ed [75] }\end{array}$ & $\begin{array}{l}\text { Tele-operated } \\
\text { robot }\end{array}$ & 5 & $\begin{array}{l}\text { Mild to severe } \\
\text { (MMSE) }\end{array}$ & At home & Yes & $\begin{array}{l}\text { Mixed method research: } \\
\text { Sequential explanatory } \\
\text { design }^{4}\end{array}$ & $50 \%$ \\
\hline 2 & $\begin{array}{l}\text { Alm et al., } 2004 \\
\text { CIRCA [35] }\end{array}$ & $\begin{array}{l}\text { Reminiscence } \\
\text { platform }\end{array}$ & $\begin{array}{l}\text { Three research } \\
\text { activities: } \\
\text { Multiple; 6; } \\
9\end{array}$ & $\begin{array}{l}\text { Mild to severe } \\
\text { (MMSE) }\end{array}$ & At home & Yes & $\begin{array}{l}\text { Qualitative research: } \\
\text { Qualitative description }\end{array}$ & $25 \%$ \\
\hline
\end{tabular}


Table 2

(Continued)

\begin{tabular}{|c|c|c|c|c|c|c|c|c|}
\hline \multirow[t]{2}{*}{ Search } & \multirow{2}{*}{$\begin{array}{l}\text { Publication Author } \\
\text { Project [Ref] }\end{array}$} & \multirow{2}{*}{$\begin{array}{l}\text { Technology } \\
\text { developed }\end{array}$} & \multicolumn{3}{|c|}{ Main characteristics of participants with dementia } & \multirow{2}{*}{$\begin{array}{l}\text { Informal } \\
\text { caregiver as } \\
\text { participant? }\end{array}$} & \multirow{2}{*}{$\begin{array}{l}\text { Research method } \\
\text { (MMAT) }\end{array}$} & \multirow{2}{*}{$\begin{array}{l}\text { Quality } \\
\text { score } \\
\text { (MMAT) }\end{array}$} \\
\hline & & & $\bar{N}=$ & $\begin{array}{l}\text { Stage of } \\
\text { dementia }\end{array}$ & $\begin{array}{l}\text { Living } \\
\text { conditions }\end{array}$ & & & \\
\hline 2 & $\begin{array}{l}\text { Van Rijn et al., } 2010 \\
\text { [37] }\end{array}$ & $\begin{array}{l}\text { Group activity } \\
\text { intervention }\end{array}$ & $\begin{array}{c}\text { Two research } \\
\text { activities: } \\
\text { group; } 12\end{array}$ & Not reported & Not reported & Yes & $\begin{array}{l}\text { Qualitative research: } \\
\text { Narrative }^{5}\end{array}$ & $50 \%$ \\
\hline 2 & $\begin{array}{l}\text { Span et al., 2015 } \\
\text { DecideGuide [49] }\end{array}$ & Website / Portal & 4 & $\begin{array}{l}\text { Mild } \\
\text { (Reisberg } \\
\text { scale) }\end{array}$ & At home & Yes & $\begin{array}{l}\text { Qualitative research: } \\
\text { Qualitative description }\end{array}$ & $100 \%$ \\
\hline 3 & $\begin{array}{l}\text { Hagethorn et al., } 2008 \\
\text { [38] }\end{array}$ & $\begin{array}{l}\text { GPS for } \\
\text { navigation }\end{array}$ & $\begin{array}{c}\text { Three research } \\
\text { activities: } \\
7,4,6\end{array}$ & Not reported & Not reported & Not clear & $\begin{array}{l}\text { Qualitative research: } \\
\text { Qualitative description }\end{array}$ & $50 \%$ \\
\hline 3 & $\begin{array}{l}\text { Cohene, Baecker \& } \\
\quad \text { Marziali, } 2005 \text { [42] }\end{array}$ & $\begin{array}{l}\text { Lifelogging } \\
\text { system }\end{array}$ & 1 & Moderate & $\begin{array}{l}\text { In nursing } \\
\text { home }\end{array}$ & Yes & $\begin{array}{l}\text { Qualitative research: Case } \\
\text { study }\end{array}$ & $75 \%$ \\
\hline 3 & $\begin{array}{l}\text { Nugent et al., } 2011 \\
\text { [76] }\end{array}$ & Reminder system & 4 & Mild (MMSE) & At home & Yes & $\begin{array}{l}\text { Qualitative research: Case } \\
\text { study }\end{array}$ & $25 \%$ \\
\hline 3 & $\begin{array}{l}\text { Alm et al., } 2005 \\
\text { CIRCA [77] }\end{array}$ & $\begin{array}{l}\text { Reminiscence } \\
\text { platform }\end{array}$ & $\begin{array}{l}\text { Two research } \\
\text { activities: } \\
40,18\end{array}$ & $\begin{array}{l}\text { Mild to severe } \\
\text { (MMSE) }\end{array}$ & $\begin{array}{l}\text { In nursing } \\
\text { home }\end{array}$ & Not clear & $\begin{array}{l}\text { Mixed methods: } \\
\text { Embedded design }\end{array}$ & $25 \%$ \\
\hline 3 & $\begin{array}{l}\text { De Beer et al., } 2010 \\
\text { [23] }\end{array}$ & $\begin{array}{l}\text { Medicine } \\
\text { dispenser }\end{array}$ & $\begin{array}{l}\text { Two different } \\
\text { activities: } \\
\text { individuals; } \\
2\end{array}$ & Not reported & At home & Yes & $\begin{array}{l}\text { Qualitative research: } \\
\text { Qualitative description }\end{array}$ & $25 \%$ \\
\hline 3 & $\begin{array}{l}\text { Morán \& Meza-Kubo, } \\
2009 \text { [78] }\end{array}$ & $\begin{array}{l}\text { Cognitive } \\
\text { stimulation }\end{array}$ & 10 & Not reported & $\begin{array}{l}\text { In nursing } \\
\text { home }\end{array}$ & No & $\begin{array}{l}\text { Qualitative research: } \\
\text { Qualitative description }\end{array}$ & $50 \%$ \\
\hline 3 & $\begin{array}{l}\text { Gibson et al., } 2016 \\
\text { [56] }\end{array}$ & $\begin{array}{l}\text { App for } \\
\text { reminiscence }\end{array}$ & 7 & Not reported & At home & Yes & $\begin{array}{l}\text { Mixed method: Case } \\
\text { report }^{6}\end{array}$ & $50 \%$ \\
\hline 3 & Wan et al., 2016 [79] & $\begin{array}{l}\text { GPS for } \\
\text { localization }\end{array}$ & 2 & Not reported & $\begin{array}{l}1 \text { in nursing } \\
\text { home }\end{array}$ & Yes & $\begin{array}{l}\text { Qualitative research: Case } \\
\text { study }\end{array}$ & $50 \%$ \\
\hline 3 & $\begin{array}{l}\text { Morrissey et al., } 2016 \\
\text { [24] }\end{array}$ & $\begin{array}{l}\text { Music } \\
\text { intervention }\end{array}$ & $\begin{array}{l}\text { Different } \\
\text { research } \\
\text { activities: } \\
\text { multiple } \\
\text { users at } \\
\text { multiple } \\
\text { sites }\end{array}$ & Not reported & $\begin{array}{l}\text { In nursing } \\
\text { home }\end{array}$ & No & $\begin{array}{l}\text { Qualitative research: } \\
\text { Ethnography }\end{array}$ & $75 \%$ \\
\hline 3 & König et al., 2016 [80] & Reminder system & 12 & $\begin{array}{l}\text { Mild to } \\
\text { moderate }\end{array}$ & $\begin{array}{l}\text { In nursing } \\
\text { home }\end{array}$ & Yes & $\begin{array}{l}\text { Qualitative research: } \\
\text { Qualitative description }\end{array}$ & $50 \%$ \\
\hline
\end{tabular}


Three research

activities:

Not reported

and groups;

8; group

Wu et al., 2016 [26

GPS for

navigation

Three research

Mild

10; several; 5

$3 \quad$ Ly et al., 2015 [27]

Sensors and

lighting cues

for indoor

navigation

3 Holbø, Bøthun \&

Dahl, 2013 [39]

navigation

3 Mayer \& Zach, 2013

[51]

Platform with

reminder

system and

communication

3 Jordan et al., 2013

[33]

Platform for

communication

Three research

activities:

several; 12 ; 4

Three research

activities: 3 ;

$3 ; 3$

Three research
activities: $5,6,5$

Moderate to

severe

Mild

Mild to

moderate

Two research

Mild to

moderate

GPS for

[17]

localization

Different

research

activities:

16,2

3 Schneider et al., 2013

[28]

Platform with

reminder

system and

communication

3 Boyd et al., 2014 [29]

Different

research

activities: 3 ,

9 , group

Three research

activities;

Mild to

moderate

Not reported

Mild to

conferencing

In nursing

home

No

Not reported

No

In nursing

home

No

At home

Yes

Not reported Yes

At home

At home and
in nursing

home

Not reported

Yes

Qualitative research:

Ethnography

$25 \%$

Qualitative research:

$25 \%$

Qualitative description

At home

Yes
Qualitative research:

Qualitative research: Case

study

Qualitative research:

Qualitative description

$25 \%$

Qualitative research: Case

study

Qualitative research:

Qualitative description

and Case study

Qualitative research: Qualitative description
Qualitative description

Qualitative research: Case 
Table 2

(Continued)

\begin{tabular}{|c|c|c|c|c|c|c|c|c|}
\hline \multirow[t]{2}{*}{ Search } & \multirow{2}{*}{$\begin{array}{l}\text { Publication Author } \\
\text { Project [Ref] }\end{array}$} & \multirow{2}{*}{$\begin{array}{l}\text { Technology } \\
\text { developed }\end{array}$} & \multicolumn{3}{|c|}{ Main characteristics of participants with dementia } & \multirow{2}{*}{$\begin{array}{l}\text { Informal } \\
\text { caregiver as } \\
\text { participant? }\end{array}$} & \multirow{2}{*}{$\begin{array}{l}\text { Research method } \\
\text { (MMAT) }\end{array}$} & \multirow{2}{*}{$\begin{array}{l}\text { Quality } \\
\text { score } \\
\text { (MMAT) }\end{array}$} \\
\hline & & & $\bar{N}=$ & $\begin{array}{l}\text { Stage of } \\
\text { dementia }\end{array}$ & $\begin{array}{l}\text { Living } \\
\text { conditions }\end{array}$ & & & \\
\hline 3 & $\begin{array}{l}\text { Donnelly et al., } 2010 \\
\text { [30] }\end{array}$ & Reminder system & $\begin{array}{l}\text { Two research } \\
\text { activities; } \\
\text { individuals, } \\
4\end{array}$ & Mild (MMSE) & At home & Yes & $\begin{array}{l}\text { Qualitative research: } \\
\text { Qualitative description }\end{array}$ & $50 \%$ \\
\hline 3 & $\begin{array}{l}\text { Nugent et al., 2008 } \\
\text { COGKNOW [81] }\end{array}$ & $\begin{array}{l}\text { Platform with } \\
\text { reminder } \\
\text { system, GPS } \\
\text { and sensors for } \\
\text { surveillance }\end{array}$ & $\begin{array}{l}\text { Two research } \\
\text { activities: } \\
17,16\end{array}$ & Mild & At home & Yes & $\begin{array}{l}\text { Qualitative research: } \\
\text { Qualitative description }\end{array}$ & $25 \%$ \\
\hline 3 & Lee \& Dey, 2007 [40] & $\begin{array}{l}\text { Lifelogging } \\
\text { system }\end{array}$ & $\begin{array}{l}\text { Two research } \\
\text { activities: } 5 \text {; } \\
5\end{array}$ & $\begin{array}{l}\text { Mild to } \\
\text { moderate }\end{array}$ & At home & Yes & $\begin{array}{l}\text { Qualitative research: } \\
\text { Ethnography and } \\
\text { qualitative description }\end{array}$ & $75 \%$ \\
\hline 3 & $\begin{array}{l}\text { Tobiasson et al., } 2015 \\
\text { [50] }\end{array}$ & $\begin{array}{l}\text { Game for physical } \\
\text { exercise }\end{array}$ & $\begin{array}{l}\text { Different } \\
\text { activities: } \\
10,12\end{array}$ & $\begin{array}{l}\text { Moderate to } \\
\text { severe }\end{array}$ & $\begin{array}{l}\text { In nursing } \\
\text { home }\end{array}$ & Yes & $\begin{array}{l}\text { Qualitative research: } \\
\text { Qualitative description }\end{array}$ & $100 \%$ \\
\hline 3 & $\begin{array}{l}\text { Huldtgren et al., } 2016 \\
\text { [31] }\end{array}$ & $\begin{array}{l}\text { Reminiscence in } \\
\text { book }\end{array}$ & $\begin{array}{l}\text { Different } \\
\text { research } \\
\text { activities: } \\
\text { groups, } 8\end{array}$ & Not reported & $\begin{array}{l}\text { In nursing } \\
\text { home }\end{array}$ & No & $\begin{array}{l}\text { Qualitative research: } \\
\text { Qualitative description }\end{array}$ & $100 \%$ \\
\hline 3 & Lazar et al. 2017 [32] & $\begin{array}{l}\text { Platform for } \\
\text { communication } \\
\text { and social } \\
\text { sharing }\end{array}$ & $\begin{array}{l}\text { Different } \\
\text { research } \\
\text { activities: } \\
\text { group and } \\
\text { individuals; } \\
2\end{array}$ & Not reported & $\begin{array}{c}\text { In nursing } \\
\text { home }\end{array}$ & Yes & $\begin{array}{l}\text { Qualitative research: } \\
\text { Ethnography and case } \\
\text { study }\end{array}$ & $100 \%$ \\
\hline
\end{tabular}

\footnotetext{
1"In-depth exploration and/or explanation of issues intrinsic to a particular case. A case can be anything from a decision-making process, to a person, an organization, or a country." [20] 2"The qualitative and quantitative components are concomitant. The purpose is to support a qualitative study with a quantitative sub-study (measures), or to better understand a specific issue of a quantitative study using a qualitative sub-study." [20]. "3here is no specific methodology, but a qualitative data collection and analysis, e.g., in-depth interviews or focus groups, and hybrid thematic analysis (inductive and deductive." [20]. " "The quantitative component is followed by the qualitative. The purpose is to explain quantitative results using qualitative findings."

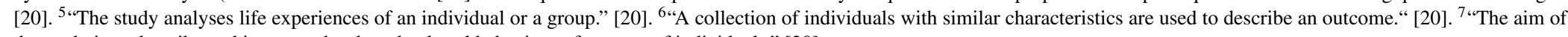
the study is to describe and interpret the shared cultural behaviour of a group of individuals." [20].
} 


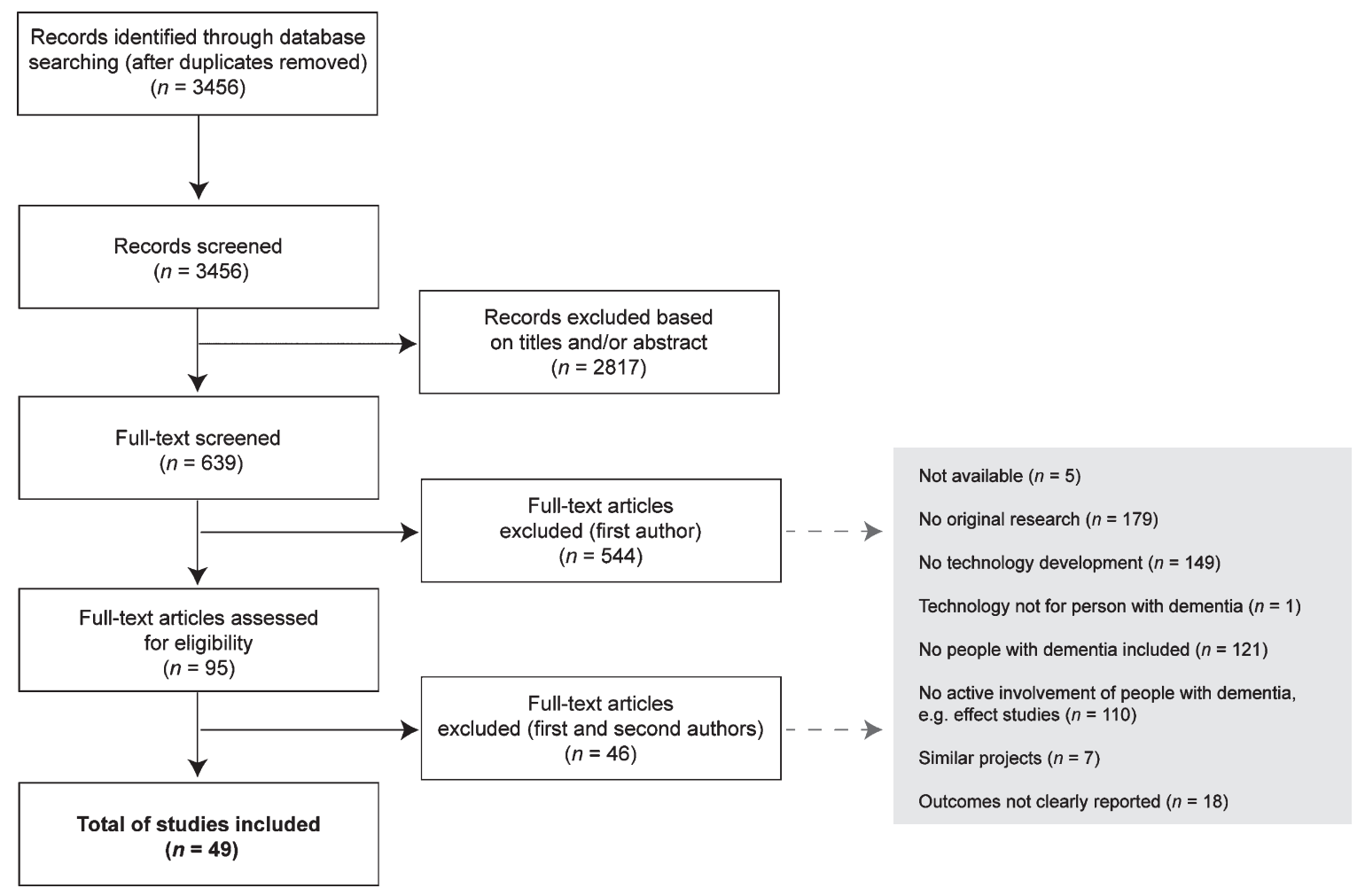

Fig. 1. Flowchart of selection process.

\section{Phase of technology development}

We discriminated between four phases in technology development: pre-design, generative, evaluative, and post-design (Fig. 2). Researchers actively involved people with dementia most often in the evaluative phase of development $(n=38)$, but also quite regularly in the generative phase $(n=25)$. The majority of the articles describing activities in this generative phase (17 out of 25) stem from the HCI and Design field (i.e., found in search 3). Studies reporting activities in the post-design phase do not include any insights from activities in earlier phases. About half of all selected articles describe research activities performed in one phase of development $(n=26)$, although they sometimes describe several research activities within this phase. For example, two articles describe multiple rounds of user testing in the evaluative phase $[35,36]$. In 20 articles, the described research activities with people with dementia occur in two phases. Often $(n=15)$ these are the generative phase and the evaluative phase. Two articles describe the involvement of people with dementia in the pre-design phase and the evaluative phase $[37,38]$ and three articles describe activities

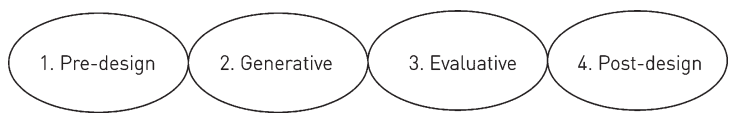

Fig. 2. Four phases of technology development.

in the pre-design phase and generative phase $[17,39$, 40]. Furthermore, in three articles the research activities cover three phases of the technology development process: pre-design, generative, and evaluative [32, $41,42]$. In several studies, activities in the other phases are described but were not conducted with people with dementia directly such as interviewing in the generative phase with (informal) carers [24, $33,37]$ and experts $[28,32]$ or brainstorming in the generative phase within the project team [38, 41]. The input of others is regarded as very relevant in the development of new technology, but multiple studies also report relatively large differences between preferences of informal caregivers and people with dementia [43-46]. Informal caregivers, for example, prioritize functionalities to monitor the safety of their relative as more important than the person with dementia themselves. 
Table 3

Variety of purposes (not mutual exclusive) of supportive technology developed in included articles

\begin{tabular}{|c|c|}
\hline Purpose of Technology & Reference numbers and Name of projects (if applicable) \\
\hline \multicolumn{2}{|l|}{ Support in daily life } \\
\hline Reminder system & {$[28,30,33,44,51,65,76,80]$ and COGKNOW project $[46,70,82]$} \\
\hline Medicine dispenser & {$[23]$} \\
\hline Robot & Ed project $[45,75]$ \\
\hline Item locator & {$[41]$ and COGKNOW project $[46,70,82]$} \\
\hline Automatic wheelchair & [73] \\
\hline Light armature & {$[55]$} \\
\hline \multicolumn{2}{|l|}{ Safety } \\
\hline Sensors for surveillance & Rosetta project $[48,54]$ and COGKNOW project $[46,70,82]$ \\
\hline Indoor navigation support system & [83] \\
\hline Outdoor localization system & {$[17,26,28,58,72,79]$ and COGKNOW project $[46,70,82]$} \\
\hline Outdoor navigation support system & {$[38,39]$} \\
\hline \multicolumn{2}{|l|}{ Meaningful activities } \\
\hline Reminiscence & {$[31,33,56]$ and CIRCA project $[35,77]$} \\
\hline Musical interventions & {$[24,25,57]$} \\
\hline Lifelogging & {$[40,42]$} \\
\hline Cognitive stimulation & {$[52,78]$} \\
\hline Physical exercise & [50] \\
\hline Group activity & [37] \\
\hline \multicolumn{2}{|l|}{ Communication } \\
\hline Video conferencing & $\begin{array}{l}{[28,29,51,71] \text { and Easy-to-use videophone project }[43,53] \text { and }} \\
\text { COGKNOW project }[46,70,82]\end{array}$ \\
\hline Sending text messages & [33] \\
\hline Social sharing & [32] \\
\hline \multicolumn{2}{|l|}{ Information } \\
\hline Informational websites & [74] and DecideGuide project $[47,49]$ \\
\hline \multicolumn{2}{|l|}{ Clinical testing } \\
\hline Cognitive screening & {$[36]$} \\
\hline
\end{tabular}

\section{Role of people with dementia}

In a limited number of articles $(n=7)$, the people with dementia were involved in multiple phases of development and were iteratively consulted to codesign the technology. Four of these articles report that the people with dementia involved were in mild to moderate stages of dementia. In all seven studies the original idea of the technology or service to be developed was already formed before the involvement of people with dementia, such as an interactive webtool [47] or an integration of three existing supportive technologies [48]. In some studies, design choices were still made by the researchers themselves. Researchers in two articles actually design different prototypes in order to let the person with dementia steer design choices [23, 42]. None of the seven co-design articles describe measurements on whether the people with dementia felt as equal partners in the project. In the remaining articles $(n=42)$, the people with dementia were involved as informant in the projects, although some studies do report that they work according the participatory design approach. Either the research described in these articles only covered one phase of development (e.g., $[46,49,50])$ or the people with dementia did not actively contribute in subsequent research activities $[37,51]$.

\section{Research methods and materials in all phases of development}

The most common methods for involving people with dementia, in all phases of development, are interviews and observations (see Table 4). In some cases, different types of elicitation material were used during the interviews (e.g., scenarios, pictures, mock-ups), but this is not standard practice. Several researchers reported problems with the interview method if no elicitation material was used, such as people with dementia having troubles answering abstract questions [52], difficulties with assessing and communicating their own experiences $[46,51]$, and giving socially desirable answers [46, 
Table 4

Different methods and materials used in design research phases of technology development

\begin{tabular}{|c|c|c|c|c|}
\hline Phase & Pre-design & Generative & Evaluative & Post-design \\
\hline Reference numbers & {$[17,32,37-42,78]$} & $\begin{array}{c}15,23,25-30,32, \\
33,35,39-42,47, \\
48,51,53,65,70, \\
72,77,79,81]\end{array}$ & $\begin{array}{l}{[23,25-27,29-32,} \\
\quad 33,35-38,41-43, \\
45-49,51,52,54-58, \\
70,71,73-77,79-81]\end{array}$ & {$[24,44,50]$} \\
\hline \multirow[t]{2}{*}{$\begin{array}{l}\text { Methods used with } \\
\text { people with dementia } \\
\text { (number of studies } \\
\text { that used this method) }\end{array}$} & $\begin{array}{l}\text { Interviews } \\
\text { Semi-structured (1) } \\
\text { Unstructured (1) }\end{array}$ & $\begin{array}{l}\text { Interviews } \\
\text { Semi-structured (5) } \\
\text { Structured (1) } \\
\text { Unstructured (1) }\end{array}$ & $\begin{array}{l}\text { Interviews } \\
\text { Semi-structured (14) } \\
\text { Structured (6) } \\
\text { Unstructured (6) }\end{array}$ & $\begin{array}{l}\text { Interviews } \\
\text { Semi-structured (2) }\end{array}$ \\
\hline & $\begin{array}{l}\text { Observations } \\
\text { Controlled (1) } \\
\text { Unstructured (2) } \\
\text { Participant (3) } \\
\text { Group sessions } \\
\text { Focus groups (1) } \\
\\
\text { Diary } \\
\text { Written (1) } \\
\text { Photo (1) }\end{array}$ & $\begin{array}{l}\text { Observations } \\
\text { Controlled (3) } \\
\text { Unstructured (4) } \\
\text { Participant (4) } \\
\text { Group sessions } \\
\text { Focus groups (8) } \\
\text { Workshops (6) } \\
\text { Personal design sessions (1) }\end{array}$ & $\begin{array}{l}\text { Observations } \\
\text { Controlled (17) } \\
\text { Unstructured (9) } \\
\text { Participant (1) } \\
\text { Group sessions } \\
\text { Focus groups (2) } \\
\text { Workshop (1) } \\
\text { Self-administered } \\
\quad \text { questionnaires } \\
\text { Standardized (5) } \\
\text { Other (4) }\end{array}$ & $\begin{array}{l}\text { Observations } \\
\text { Participant (2) }\end{array}$ \\
\hline $\begin{array}{l}\text { Materials used (number } \\
\text { of studies that used } \\
\text { this material) }\end{array}$ & $\begin{array}{l}\text { Neck worn cameras } \\
\text { (1) }\end{array}$ & $\begin{array}{l}\text { Neck worn cameras (1) } \\
\text { Scenarios (6) } \\
\text { Mock-ups } \\
\text { Sketches and digital images (4) } \\
\text { Paper prototypes (5) } \\
\text { Tangible objects (4) } \\
\text { Probes and other prompts } \\
\text { Personal photos (2) and other } \\
\quad \text { pictures (4) } \\
\text { Existing technology (4) } \\
\text { Lego (1) } \\
\text { Games (1) } \\
\text { Creative material; foam, } \\
\quad \text { post-its (1) }\end{array}$ & $\begin{array}{l}\text { Prototypes } \\
\text { Wizard-of-Oz prototype (4) } \\
\text { Alpha prototype (6) } \\
\text { Beta prototype (27) } \\
\text { Fully functional prototype (3) } \\
\text { Probes and other prompts } \\
\text { Technology (1) } \\
\text { Probekit/game (1) }\end{array}$ & $\begin{array}{l}\text { Finished product (2) } \\
\text { Probes and other prompts } \\
\text { All sorts; different music } \\
\text { instruments, sheets of } \\
\text { material etc. (1) }\end{array}$ \\
\hline
\end{tabular}

53]. In the reviewed articles, observations were usually conducted when people were invited to test a beta-prototype in the evaluation phase $(n=27)$. Some researchers elucidate this by stating that people with dementia cannot imagine how a non-functional prototype is supposed to work [52] or have trouble comprehending low fidelity prototypes [47]. On the other hand, downsides of using functional prototypes are shared in the studies as well such as people with dementia being afraid of breaking the technology [51], timing issues with installation procedures [54], and technical failures affecting the attitude toward the system [30, 49]. Other researchers also report using low fidelity prototypes, such as paper prototypes, Wizard-of-Oz prototypes, or alpha prototypes. Paper prototypes were mainly used as a representation of the final product, instead of being used during a co-design activity. One article describes a design research activity with creative materials, such as foam and Lego, used to generate ideas for the technology under development [39].
In general, authors provide only a limited rationale as to why they choose certain research methods and materials. Two studies researched the technology as well as the used methods and materials $[55,56]$. Both studies argue for minimizing the need for retrospective reflection in a research activity with people with dementia.

\section{Impact of involvement on technology and participants}

The impact of the involvement of people with dementia on technology development was identified in terms of changes to the technology and in terms of experiences of the participants themselves (Table 5). Out of the total number of 49 included articles, 11 articles did not report directly applied changes as a result of users' involvement, in 19 articles one change was made to the technology and in 19 articles multiple changes were attributed to the input of participants with dementia. As expected, the type of changes dif- 
Table 5

Role of participants with dementia and the impact of this role on technology and experience of participation

\begin{tabular}{|c|c|c|c|c|c|}
\hline $\begin{array}{l}\text { Publication } \\
\text { Author } \\
\text { Project }\end{array}$ & $\begin{array}{l}\text { Design } \\
\text { approach }\end{array}$ & $\begin{array}{l}\text { Role of } \\
\text { person with } \\
\text { dementia } \\
\text { Informant } \\
\text { Co-designer }\end{array}$ & 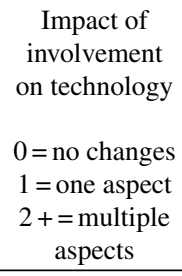 & $\begin{array}{c}\text { Impact of } \\
\text { involvement } \\
\text { on person with } \\
\text { dementia } \\
-=\text { negative } \\
\text { impact } \\
+=\text { positive } \\
\text { impact } \\
\end{array}$ & \\
\hline $\begin{array}{l}\text { Karlsson et al., } \\
\quad 2011 \\
\text { COGKNOW [70] }\end{array}$ & $\begin{array}{l}\text { User-driven } \\
\text { design }\end{array}$ & Informant & 0 & + & $\begin{array}{l}\text { Participants were relaxed, despite being observed. Interviews at the homes of the } \\
\text { participants ensured familiar environments. }\end{array}$ \\
\hline $\begin{array}{l}\text { Span et al. } 2017 \\
\text { DecideGuide [47] }\end{array}$ & $\begin{array}{l}\text { Participatory } \\
\text { design }\end{array}$ & Co-designer & $2+$ & $-\&+$ & $\begin{array}{l}\text { People with dementia were asked why they participated. Reasons were (1) to be useful by } \\
\text { contributing to research activities, (2) to contribute to a better quality of life for future } \\
\text { dementia patients, and (3) give one's opinion. Researchers report no signs of distress } \\
\text { during interviews. Most participants enjoyed in sharing their experiences, although } \\
\text { talking about some topics was emotional. }\end{array}$ \\
\hline $\begin{array}{l}\text { Meiland et al. } \\
2012 \\
\text { COGKNOW [46] }\end{array}$ & $\begin{array}{l}\text { Participatory } \\
\text { design }\end{array}$ & Informant & 0 & Not described & $\begin{array}{l}\text { The expectations of the users regarding the device to be developed could not always be } \\
\text { met. Time constraints limited the ability to develop all functionalities suggested by } \\
\text { users who participated in more than one cycle. }\end{array}$ \\
\hline $\begin{array}{l}\text { Lopes et al. } 2016 \\
\text { [41] }\end{array}$ & $\begin{array}{l}\text { Action } \\
\text { research }\end{array}$ & Co-designer & 1 & + & $\begin{array}{l}\text { People with dementia asked for solutions that would be easy to use or that could be } \\
\text { integrated in a more global assistive solution (personal robot, mobile phone...). } \\
\text { People with dementia were empowered because of their participation in all phases of } \\
\text { development, including requirement determination and prototype testing. }\end{array}$ \\
\hline $\begin{array}{l}\text { Boman et al. } 2014 \\
\text { [43] }\end{array}$ & $\begin{array}{r}\text { Inclusive } \\
\text { design }\end{array}$ & Informant & $2+$ & + & $\begin{array}{l}\text { People with dementia pointed out that it should be possible to adjust the features of the } \\
\text { videophone to each individual's needs and wishes. Other functionalities were also } \\
\text { reviewed. Albeit facing some problems in the test sessions, the participants with } \\
\text { dementia strongly expressed positive feedback and appreciation of being involved. }\end{array}$ \\
\hline $\begin{array}{l}\text { Wang et al., } 2017 \\
\text { Ed [45] }\end{array}$ & Not reported & Informant & 1 & Not described & People with dementia report the need for adaptability of the technology. \\
\hline $\begin{array}{l}\text { Peeters et al., } \\
2016[57]\end{array}$ & $\begin{array}{l}\text { Situated } \\
\text { Cognitive } \\
\text { Engineering }\end{array}$ & Informant & $2+$ & - & $\begin{array}{l}\text { People with dementia contributed to refinement of research rationale (including new } \\
\text { structure of app, linking to personal life events, adding music, adding new pictures) } \\
\text { Researchers observed that the people with dementia became very tired, less talkative, or } \\
\text { even withdrawn towards the end of the test session. }\end{array}$ \\
\hline $\begin{array}{l}\text { Meiland et al., } \\
2014 \\
\text { ROSETTA [48] }\end{array}$ & $\begin{array}{l}\text { Participatory } \\
\text { design }\end{array}$ & Co-designer & $2+$ & Not described & $\begin{array}{l}\text { People with dementia discussed functionalities and gave remarks on interface design } \\
\text { issues (including icon design, amount of information, content). }\end{array}$ \\
\hline $\begin{array}{l}\text { Suijkerbuijk et al., } \\
2015 \text { [55] }\end{array}$ & Not reported & Informant & 1 & $-\&+$ & $\begin{array}{l}\text { Comments about the aesthetics of the light armature were provided. People with dementia } \\
\text { did not report any discomfort of participating in this study. Several aspects appeared to } \\
\text { influence their feelings of involvement and motivation: reminding of loss of abilities; } \\
\text { feeling of fulfilment; research activity was fun. }\end{array}$ \\
\hline
\end{tabular}




\begin{tabular}{|c|c|c|c|c|}
\hline $\begin{array}{l}\text { Olsson et al., } 2013 \\
\text { [58] }\end{array}$ & Not reported & Informant & 0 & $-\&+$ \\
\hline $\begin{array}{c}\text { Boman et al., } \\
2014 \text { [53] }\end{array}$ & $\begin{array}{r}\text { Inclusive } \\
\text { design }\end{array}$ & Informant & $2+$ & Not described \\
\hline $\begin{array}{l}\text { Kerkhof et al., } \\
2015 \text { [44] }\end{array}$ & $\begin{array}{l}\text { User-centered } \\
\text { design }\end{array}$ & Informant & $2+$ & + \\
\hline $\begin{array}{l}\text { Hattink et al., } \\
2016\end{array}$ & Not reported & Informant & 0 & Not described \\
\hline ROSETTA [54] & & & & \\
\hline $\begin{array}{l}\text { Pakrasi et al., } \\
2015 \text { [71] }\end{array}$ & Not reported & Informant & 0 & Not described \\
\hline $\begin{array}{l}\text { Dethlefs et al., } \\
2017 \text { [52] }\end{array}$ & Not reported & Informant & 0 & Not described \\
\hline $\begin{array}{l}\text { Jacova et al., } 2015 \\
\text { [36] }\end{array}$ & Not reported & Informant & 1 & Not described \\
\hline $\begin{array}{l}\text { McCabe \& Innes, } \\
2013 \text { [72] }\end{array}$ & Not reported & Informant & 1 & + \\
\hline $\begin{array}{l}\text { Wang et al., } 2011 \\
\text { [73] }\end{array}$ & Not reported & Informant & 1 & Not described \\
\hline $\begin{array}{l}\text { Hattink et al., } \\
2016 \text { [74] }\end{array}$ & Not reported & Informant & 1 & Not described \\
\hline $\begin{array}{l}\text { Wolters et al., } \\
2016 \text { [65] }\end{array}$ & Not reported & Informant & $2+$ & Not described \\
\hline $\begin{array}{l}\text { Begum et al., } 2013 \\
\text { Ed [75] }\end{array}$ & $\begin{array}{l}\text { User-centered } \\
\text { design }\end{array}$ & Informant & $2+$ & Not described \\
\hline $\begin{array}{l}\text { Alm et al., } 2004 \\
\text { CIRCA [35] }\end{array}$ & Not reported & Informant & $2+$ & Not described \\
\hline
\end{tabular}

Some persons with dementia made statements about desired changes in the design of the technology, but not further defined. Despite the emotional upheaval of describing and reflecting on their situation, all of the participants (both persons with dementia and their spouses) appreciated being given an opportunity to speak with someone who showed an interest in hearing their stories.

People with dementia opted for more flexibility in design and functionalities. The videophone should be modern and attractive. Also, ideas about introducing the system to new users were shared.

Issues were mentioned regarding the implementation (installation errors, inefficient use) and needs for further development (representation of content). The authors report that some residents actually liked being able to contribute and that their input mattered, and that it was remarkable to see how articulate the people with dementia were about their needs.

This evaluation study showed that the Rosetta system was anticipated very useful by the participants, especially when the major technical problems would be resolved.

Participants reporting greater autonomy, less isolation, and improved quality of life.

This study investigates the general acceptability of computer-based cognitive stimulation.

People with dementia disliked lengthy written instructions, and asked for step-by-step instructions with pictorial support. Other interface issues, such as busy screen layout and consistency, were resolved.

People with dementia shared many insights on appropriate design for GPS systems. Participants expressed their enjoyment of taking part

In the design of future prototypes, further attention needs to be paid to size, form, and construction and the interaction of exterior features with how the device is perceived.

People with dementia had most troubles with reaching and accessing the site (e.g., logging in, typing the URL).

General attitude towards cognitive assistants, timing of prompts, voice and interface preferences. Personalization of system is crucial.

The developer will focus on social interactive aspect of the robot in future development based on this study. Furthermore, they intend to perform a few changes to the overall appearance of the robot which include using an animated avatar for speech delivery and using a low pitch female voice.

People with dementia comment on design issues (such as contrast of screen, size of the typeface, the brightness of the visual images) but also reported on the selection of stimuli available. 
Table 5

Role of participants with dementia and the impact of this role on technology and experience of participation

\begin{tabular}{|c|c|c|c|c|c|}
\hline $\begin{array}{l}\text { Publication } \\
\text { Author } \\
\text { Project }\end{array}$ & $\begin{array}{l}\text { Design } \\
\text { approach }\end{array}$ & $\begin{array}{c}\text { Role of } \\
\text { person with } \\
\text { dementia } \\
\text { Informant } \\
\text { Co-designer }\end{array}$ & $\begin{array}{l}\text { Impact of } \\
\text { involvement } \\
\text { on technology } \\
0=\text { no changes } \\
1=\text { one aspect } \\
\begin{array}{c}2+=\text { multiple } \\
\text { aspects }\end{array}\end{array}$ & $\begin{array}{l}\text { Impact of } \\
\text { involvement } \\
\text { on person with } \\
\text { dementia } \\
-=\text { negative } \\
\text { impact } \\
+=\text { positive } \\
\text { impact } \\
\end{array}$ & \\
\hline $\begin{array}{l}\text { Van Rijn et al., } \\
2010[37]\end{array}$ & $\begin{array}{l}\text { Participatory } \\
\text { design }\end{array}$ & Informant & $2+$ & + & $\begin{array}{l}\text { Interactions with the people with dementia led to the decision of not using laptop screens } \\
\text { in the design. During user tests, the design team report that triggers are needed for } \\
\text { people with dementia to start using the system. The people with dementia involved in } \\
\text { the testing really enjoyed participating in the design project and the conversations they } \\
\text { had with the team members. The design team was not only gathering design } \\
\text { information but became providers of activities as well. Four months after the design } \\
\text { project, the care professionals reported that some people still asked about the designers. }\end{array}$ \\
\hline $\begin{array}{l}\text { Span et al., 2015 } \\
\text { DecideGuide [49] }\end{array}$ & $\begin{array}{l}\text { Participatory } \\
\text { design }\end{array}$ & Informant & 1 & Not described & $\begin{array}{l}\text { People with dementia commented on navigation and user friendliness of the interface } \\
\text { (such as text and buttons needed to be enlarged. }\end{array}$ \\
\hline $\begin{array}{l}\text { Hagethorn et al., } \\
2008[38]\end{array}$ & $\begin{array}{l}\text { User-centered } \\
\text { design }\end{array}$ & Informant & $2+$ & Not described & $\begin{array}{l}\text { People with dementia were able to steer the design by commenting on important } \\
\text { functionalities (e.g., being able to make a "free" walk, alarm function) and interface } \\
\text { issues (few buttons, interface elements must be big). }\end{array}$ \\
\hline $\begin{array}{l}\text { Cohene, Baecker } \\
\quad \& \text { Marziali, } \\
2005 \text { [42] }\end{array}$ & $\begin{array}{l}\text { User-centered } \\
\text { design }\end{array}$ & Co-designer & $2+$ & Not described & $\begin{array}{l}\text { During a reminiscence session, the content of the digital life story book was established. } \\
\text { Very different prototypes were shown to the participant to inform next iterations of the } \\
\text { design. }\end{array}$ \\
\hline $\begin{array}{l}\text { Nugent et al., } \\
2011[76]\end{array}$ & Not reported & Informant & 0 & Not described & The study resulted in insights into the usage of video based reminding technology. \\
\hline $\begin{array}{l}\text { Alm et al., } 2005 \\
\text { CIRCA [77] }\end{array}$ & Not reported & Informant & 1 & Not described & $\begin{array}{l}\text { Further development will be based on the lessons learned in the evaluations; e.g., by } \\
\text { making the system more modular. }\end{array}$ \\
\hline $\begin{array}{l}\text { De Beer et al., } \\
2010[23]\end{array}$ & Not reported & Co-designer & $2+$ & Not described & $\begin{array}{l}\text { People with dementia explained the troubles with medicine intake and what they like } \\
\text { about existing systems. They reflected on a first design idea, by adding the need for a } \\
\text { portable system. }\end{array}$ \\
\hline $\begin{array}{l}\text { Morán \& } \\
\text { Meza-Kubo, } \\
2009[78]\end{array}$ & Not reported & Informant & $2+$ & Not described & $\begin{array}{l}\text { A large set of design-insights is based on the user research, including how a session with } \\
\text { the tele-assistant should be managed, how interaction with material should be } \\
\text { established etc. }\end{array}$ \\
\hline $\begin{array}{l}\text { Gibson et al., } \\
2016[56]\end{array}$ & Not reported & Informant & 0 & Not described & $\begin{array}{l}\text { People with dementia had troubles verbalizing thoughts while simultaneously testing the } \\
\text { app ('thinking aloud'). }\end{array}$ \\
\hline
\end{tabular}




\begin{tabular}{|c|c|c|c|c|c|}
\hline $\begin{array}{l}\text { Wan et al., } 2016 \\
\text { [79] }\end{array}$ & $\begin{array}{l}\text { User-centered } \\
\text { design }\end{array}$ & Informant & 0 & Not described & $\begin{array}{l}\text { Researchers describe the difficulties with involving people with dementia and translating } \\
\text { their needs into (fixed) design. }\end{array}$ \\
\hline $\begin{array}{l}\text { Morrissey et al., } \\
2016[24]\end{array}$ & $\begin{array}{l}\text { Experience- } \\
\text { centered } \\
\text { design }\end{array}$ & Informant & 1 & + & $\begin{array}{l}\text { The materials of the batons were changed according to the responses of people with } \\
\text { dementia. The interactions with the participants show that they felt free in interacting } \\
\text { with each other and the research and that they enjoyed getting a voice. }\end{array}$ \\
\hline $\begin{array}{l}\text { König et al., } 2016 \\
\text { [80] }\end{array}$ & Not reported & Informant & $2+$ & Not described & $\begin{array}{l}\text { People with dementia opted for personalization in the avatar, respond to the style of } \\
\text { prompts given and discussed that they did not want to use the system when it would } \\
\text { make them feel old. }\end{array}$ \\
\hline $\begin{array}{r}\text { Bennett, Hinder \& } \\
\text { Cater, } 2016[25]\end{array}$ & Not reported & Informant & 0 & Not described & $\begin{array}{l}\text { A design process is described but reported feedback from people with dementia was only } \\
\text { positive and did not lead to changes. }\end{array}$ \\
\hline $\begin{array}{l}\text { Wu et al., } 2016 \\
\text { [26] }\end{array}$ & Not reported & Informant & 1 & Not described & $\begin{array}{l}\text { Designers found some difficulties with the auditory cues and want to improve this in } \\
\text { further developments. }\end{array}$ \\
\hline $\begin{array}{l}\text { Ly et al., } 2015 \\
\quad[27]\end{array}$ & $\begin{array}{l}\text { People- } \\
\text { centered } \\
\text { design }\end{array}$ & Informant & 1 & Not described & $\begin{array}{l}\text { People with dementia had their saying in the design of the guiding cues; the ambient LED } \\
\text { light and the color of the light. }\end{array}$ \\
\hline $\begin{array}{l}\text { Holbø, Bøthun \& } \\
\text { Dahl, } 2013 \text { [39] }\end{array}$ & $\begin{array}{l}\text { Participatory } \\
\text { design }\end{array}$ & Co-designer & $2+$ & + & $\begin{array}{l}\text { In individual participatory design sessions, people with dementia prioritize functions of } \\
\text { the system. Most important was the insights given in the conceptual model of the final } \\
\text { design. However, researchers also report that design suggestions from the workshops } \\
\text { were not directly translated into requirements. Furthermore, researchers indicate that } \\
\text { people were empowered (no measurements reported). }\end{array}$ \\
\hline $\begin{array}{l}\text { Mayer \& Zach, } \\
\quad 2013[51]\end{array}$ & $\begin{array}{l}\text { Participatory } \\
\text { design }\end{array}$ & Informant & $2+$ & + & $\begin{array}{l}\text { Authors report many design implications from their user research, such as the use of } \\
\text { familiar items, simple and redundant interface design, explanatory pictures and videos. } \\
\text { The use of games in their research created enjoyment during focus groups and relaxed } \\
\text { atmosphere to discuss different topics. }\end{array}$ \\
\hline $\begin{array}{l}\text { Jordan et al., } 2013 \\
\text { [33] }\end{array}$ & $\begin{array}{l}\text { User-centered } \\
\text { design }\end{array}$ & Informant & 1 & + & $\begin{array}{l}\text { Most changes to the design (and added functionalities) stem from the input of carers. Only } \\
\text { redesign of the clock was based on finding from report of people with dementia. Due to } \\
\text { the setting, researchers report that people felt trusted, calm and willingly to participate. }\end{array}$ \\
\hline $\begin{array}{l}\text { Lindsay et al., } \\
\quad 2012 \text { [17] }\end{array}$ & $\begin{array}{l}\text { Participatory } \\
\text { design }\end{array}$ & Co-designer & $2+$ & $-\&+$ & $\begin{array}{l}\text { Participants with dementia were involved in multiple phases, impacting the functionalities } \\
\text { of the device and the design. Authors report on disempowering effects (when taking } \\
\text { decision based on insights), quality relationship between researchers and participants } \\
\text { (although this might have resulted in less critical feedback) and some fixation on certain } \\
\text { design issues that were not feasible to actual implement. }\end{array}$ \\
\hline $\begin{array}{l}\text { Schneider et al., } \\
2013 \text { [28] }\end{array}$ & $\begin{array}{l}\text { User-centered } \\
\text { design }\end{array}$ & Informant & 1 & Not described & $\begin{array}{l}\text { New functionalities were created by input from participants with dementia in the second } \\
\text { iteration. }\end{array}$ \\
\hline
\end{tabular}

(Continued) 
Table 5

Role of participants with dementia and the impact of this role on technology and experience of participation

\begin{tabular}{|c|c|c|c|c|c|}
\hline $\begin{array}{l}\text { Publication } \\
\text { Author } \\
\text { Project }\end{array}$ & $\begin{array}{l}\text { Design } \\
\text { approach }\end{array}$ & $\begin{array}{c}\text { Role of } \\
\text { person with } \\
\text { dementia } \\
\text { Informant } \\
\text { Co-designer }\end{array}$ & $\begin{array}{c}\text { Impact of } \\
\text { involvement } \\
\text { on technology } \\
\begin{array}{c}0=\text { no changes } \\
1=\text { one aspect } \\
2+=\text { multiple } \\
\text { aspects }\end{array}\end{array}$ & $\begin{array}{l}\text { Impact of } \\
\text { involvement } \\
\text { on person with } \\
\text { dementia } \\
-=\text { negative } \\
\text { impact } \\
+=\text { positive } \\
\text { impact }\end{array}$ & \\
\hline $\begin{array}{l}\text { Boyd et al., } 2014 \\
\text { [29] }\end{array}$ & $\begin{array}{l}\text { User-centered } \\
\text { design }\end{array}$ & Informant & 1 & Not described & $\begin{array}{l}\text { After a first testing of multiple prototypes, the people with dementia could opt for the } \\
\text { most preferred version to be further developed and tested. }\end{array}$ \\
\hline $\begin{array}{l}\text { Donnelly et al., } \\
2010[30]\end{array}$ & Not reported & Informant & 1 & Not described & Participants with dementia commented on the size and sound of the device. \\
\hline $\begin{array}{l}\text { Nugent et al., } \\
2008\end{array}$ & Not reported & Informant & 1 & Not described & $\begin{array}{l}\text { People with dementia expressed their need for different forms of personalization in } \\
\text { interface design (colors and size of buttons/fonts). }\end{array}$ \\
\hline COGKNOW [81] & & & & & \\
\hline $\begin{array}{l}\text { Lee \& Dey, } 2007 \\
\quad[40]\end{array}$ & $\begin{array}{l}\text { User-centered } \\
\text { design }\end{array}$ & Informant & 1 & Not described & $\begin{array}{l}\text { Photos were taken and sorted by people with dementia in order to define the type of cues } \\
\text { needed for the system. }\end{array}$ \\
\hline $\begin{array}{l}\text { Tobiasson et al., } \\
2015[50]\end{array}$ & $\begin{array}{l}\text { Participatory } \\
\text { design }\end{array}$ & Informant & $2+$ & Not described & $\begin{array}{l}\text { The types of game (competition) and duration of play was decided by the people with } \\
\text { dementia. Later in the process, the console was redesigned together. }\end{array}$ \\
\hline $\begin{array}{l}\text { Huldtgren et al., } \\
\quad 2016[31]\end{array}$ & $\begin{array}{l}\text { Research- } \\
\text { through- } \\
\text { design }\end{array}$ & Informant & 1 & Not described & $\begin{array}{l}\text { Many design implications were brought in by reflections from carers. One aspect coming } \\
\text { directly from participants with dementia is related to the redesign of buttons. }\end{array}$ \\
\hline $\begin{array}{l}\text { Lazar et al. } 2017 \\
\text { [32] }\end{array}$ & Not reported & Informant & 0 & Not described & $\begin{array}{l}\text { People with dementia are involved in evaluation but no direct design implications are } \\
\text { reported. }\end{array}$ \\
\hline
\end{tabular}


fered according to the phase of the development in which the input of people with dementia was collected. In the earlier phases, participants could have an influence on the conceptual idea and rationale $[39,57]$ and the functionalities of the system (e.g., [48]), while in the later phases the changes were more related to interface design (e.g., [35, 49] or implementation issues [44]. It was not always clear whether the reported changes were actually further developed in the remainder of the project.

The vast majority of the articles do not describe how participants experience their involvement (34 out of 49). However, three studies of the 15 studies that do elaborate on the experiences of participants specifically asked people with dementia about their involvement [43, 47, 55]. The remaining studies $(n=12)$ report experiences without quotes and/or reference to how these experiences were gathered. Positive experiences include feelings of fulfillment, enjoyment, and empowerment. Also, appreciation for their involvement and a relaxed and trusting interaction with researchers were identified as positive aspects of involvement. Few negative experiences of participating were reported, including becoming emotional when talking about their situation $[47,55$, 58] and feeling extremely tired during the research activity [57]. Disempowering effects were reported when design changes could not be implemented in the further development [17].

\section{DISCUSSION}

The increasing number of people with dementia worldwide creates a need for meaningful support in independent living and overall well-being in daily life. Also, the possibilities that technology afford, increase enormously with the continuous worldwide development of digitalization. Earlier work emphasizes the importance of involving people with dementia in the development of meaningful supportive or assistive technologies [11-14]. This systematic literature review updates and builds on the existing knowledge about the active involvement of people with dementia, by including a contemporary and relevant perspective. Ultimately a selection of 49 out of 3465 articles was made based on a search in seven different search databases.

Our review reveals that in the majority of the studies, research activities were mainly performed in one phase of technology development, often being the evaluative phase. Notably, the majority of the arti- cles describing activities in the generative phase stem from the HCI and Design field. Because most studies $(n=42)$ involve the people with dementia in only one phase, their role is usually limited to being an informant. It seems especially complicated to allow people in the more advanced stages of dementia an active role as equal partner over the course of a project that might easily take several years. Merely seven studies gave the people with dementia a role as a co-designer in more than one phase of the development. However, without explicit confirmation of people feeling like being treated as equal partner during the process, the extent to which they actually actively participated in a design is debatable. Our review also highlights that the most frequently used research methods were interviews and observations. Prototypes of the technology, with different levels in functioning, were used in the evaluative phase of the development process. However, the arguments for the chosen research methods and the research materials, such as prototypes, were not extensively reported in the included articles. This is problematic because this information can be valuable for future research and earlier research indicates that people with dementia might get distressed when testing only partially functioning prototypes $[28,59]$. Nevertheless, this literature review reflects that people with dementia can influence the development of technology in regards to content, design, and even the initial idea, although the impact on how they experience their own involvement remains largely unknown. Within these conclusions, "people with dementia" are treated as a homogenous group, while we are well aware that the above-mentioned insights will be different for people in various stages or types of dementia.

\section{The value of an interdisciplinary perspective}

Our review shows that including search words and databases from the field of $\mathrm{HCI}$ and Design results in finding more relevant studies involving people with dementia. Lately, there is indeed an increasing attention in the HCI community on design with people with dementia [60]. They argue that the HCI perspective can move the research focus away from only considering medical concerns toward a broader view into context, values, and the situatedness of technology use. In the development of supportive technologies for people with dementia, the HCI and Design articles often describe design-oriented studies in the generative phase or small-scale feasibility studies in the later evaluative phase of technology 
development. By including these studies in the current review, we accomplished a broader view on how and when people with dementia can be involved in technology development. Furthermore, researchers from HCI and Design are familiar with using different elicitation materials (such as scenarios and mockups) in research activities which is reported to be helpful to get an empathic understanding of the user group and their everyday lives.

However, in line with a review in self-care technologies [61], the majority of the articles from HCI and Design seem to lack a thorough theoretical grounding. In general, the methodological quality of the included studies was difficult to assess and differed, mainly because articles do not always describe research questions and analysis with sufficient detail. The expertise from HCI and Design to iteratively co-design human-centered technologies and include people with dementia in earlier phases of development should be accompanied with the expertise from the healthcare researchers on longitudinal studies. This way we ensure that the co-designed technologies eventually impact the lives of a larger group of people with dementia. Insights in appropriate research methods and materials in earlier phases of development can potentially be useful in the later evaluative and post-design phases as well. In accordance with the broader field of digital health interventions [62], we recognize that the different abovementioned competences seem to slightly converge in the field of supportive technology development for people with dementia but are, at this moment, still merely separate worlds. However, this interdisciplinary approach should be more employed in future research to combine the strengths of both worlds to fully overcome the challenges of actively involving people with dementia in the development of well suiting technologies.

\section{The challenge of early involvement of and co-design with people with dementia}

User involvement has positive effects on the development of accessible and meaningful technology. It is, however, not an easy task when the users are people living with cognitive impairments such as dementia. General lessons from projects developing supportive technology are well shared such as the importance of establishing a proper researchparticipant relationship, having dementia expertise within the multidisciplinary team, spending time in informing the participant about the research and orga- nizing a respectful exit-strategy on what to expect after participating in the project [63,64]. Nevertheless, our work shows that there is still a lack of specific knowledge on co-design methodologies for people with dementia in supportive technology development, even when including articles from the field of HCI and Design.

The reviewed articles report challenges including people with dementia in technology development because they can face difficulties answering abstract questions and communicating their own experiences over time. Informal caregivers can play an important supportive role in these activities, but cannot substitute for the involvement of people with dementia themselves since they sometimes also have different needs and opinions. The fact that most studies report on involvement of people with dementia in activities in the evaluative phase might be expected. When testing a prototype with someone with dementia, the behavior and interaction can be observed and measured, and this person can reflect on their experiences while using it. Therefore, there is less need for retrospection. It will become problematic when people with dementia are asked to reflect on their experiences over a longer period of time without the researcher in the same room. Furthermore, in the earlier phases of development (pre-design or generative) involving someone with dementia might be more challenging because the research activities require more abstract thinking [17] and interaction measures are less suitable or even impossible in the pre-design phase. To make the earlier development phases recognizable and more concrete, researchers use materials such as scenarios (e.g., [28,48,51,65]). However, the use of scenarios still needs some sort of capability to translate the scenario to someone's own life which can be difficult for someone with dementia. Moreover, researchers also use existing technology for people with dementia to evaluate and further requirements are based on the experiences people could formulate with these existing products $[17,29,41,53]$. This shows that the more concrete the research materials are, the easier it potentially becomes for people with dementia to articulate their opinion about these materials. Yet, the level of openness of the material also defines the impact the person with dementia has on the eventual development of the technology. Generative tools, such as drawing and building paper prototypes, are needed to gain new insights and share plans among the entire design team [15]. Reflecting on existing technology does not directly allow for generating ideas 
that might entail totally different (non-technological) solutions.

Despite the expansion of the current review to the field of HCI and Design, we found only a relatively small number of articles including people with dementia as actual co-designers. We expect that this role can be beneficial, but it is still not possible to draw firm conclusions on the impact of a co-design role on the technology development. We only identified one paper that uses creative design material with people with mild dementia [39]. This highlights the limited knowledge we have on how to use the creative capacity of people with dementia and actively involve them in the early phases of technology development to steer the design agenda and truly co-design supportive technologies. So, given that the original idea of the technology or service to be developed was often already formed before the involvement of people with dementia, this raises the question whether the field is at this point still more technology driven instead of user driven. This might be a fundamental reason why the uptake of technology by people with dementia is currently so limited.

\section{Strengths and limitations}

Next to the abovementioned value of expanding our search in multiple ways including expansion to our search to the field of HCI and Design, our results show that there is an increased interest in involving people with dementia in the development of supportive technologies. This demonstrates the relevance of the current review to update the existing body of knowledge. Furthermore, the strengths of the current review are also in the rigor of the methodology, by independently screening a high number of abstracts, titles, and full papers by multiple researchers. Additionally, by using earlier work for the search strategy, this review builds on existing knowledge which is highly needed in this field [66]. The extension of the search key words and search databases resulted in a list of articles that, to our beliefs, include all relevant studies on design and technology development for people with dementia available in the scientific literature.

Nevertheless, the following limitations have to be considered. Although we attempted to objectively rate the articles on different criteria, we acknowledge that criteria could only be assessed based on available descriptions in the article and then subjectively assessed (e.g., the impact of the involvement of people with dementia on the technology devel- oped). Similar problems are also mentioned in related work [12]. However, a structured approach was followed and whenever there was disagreement between both raters, consensus was reached by discussion. Due to our scope of including articles that report on 1) active involvement of people with dementia and 2) technology development, we might have limited the insights from studies such as the creative work with people with dementia living in care facilities that did not involve technology development $[67,68]$. This might have had given some additional insights in useful research methods and materials. In particular, we recognize that need elicitation studies, without clear identification of being part of technology development, are now not included in this review. Given the large number of articles found within the current scope, the value of focusing our search seemed necessary. Our results, therefore, present insights for the specific field of developing supportive technologies for people with dementia.

\section{Conclusion}

This review reveals that there is a growing amount of research projects in order to develop supportive technologies for people with dementia (e.g., within the European AAL Program). Compared to earlier work [12], there is an increase in studies eliciting an active involvement of people with dementia. By adding studies from the HCI and Design fields, the current review has outlined a more balanced view on where in the development process people with dementia can be involved. Not only are people with dementia able to evaluate technology, they can also play an important role in the generative phase of development to steer the design of supportive technology. Involving people with dementia as co-designers in the development of technology is deemed challenging, but not impossible. However, even with the update and expansion in the current review, specific insights in appropriate research methods and materials is still lacking. Extensive reports on the methodology and evaluation of the experiences of involvement of the people with dementia themselves are generally missing. This makes appreciation and further improvement of the active involvement and co-design difficult within this interdisciplinary field. Especially since some of the current developments can be regarded as more technology-driven instead of user-driven, our results emphasize the importance of a better understanding of how people with dementia can and ought to be involved. Future research 
should therefore continue to focus on and describe how research methods and materials could be shaped, chosen and applied. This does not need to interfere with the explorative nature of design activities when writing detailed method stories of these design activities with different people with dementia [18]. In line with person-centered dementia care [69], the research methods and materials should consider users' changing individual strengths and vulnerabilities, and give people with dementia their desired role in the development of supportive technology. Only when people with dementia have the right sense of control, the gained empathic understanding of this user group by designers, developers and researchers will result in more meaningful and suitable technology.

\section{ACKNOWLEDGMENTS}

Vilans, Centre of Expertise for long-term care, initiated and financed this study. We greatly acknowledge the support of Dirk Lukkien, Nick Zonneveld, Cynthia Hofman, and Amber van Geerestein in reviewing earlier versions of this manuscript.

Authors' disclosures available online (https:// www.j-alz.com/manuscript-disclosures/19-0050r1).

\section{SUPPLEMENTARY MATERIAL}

The supplementary material is available in the electronic version of this article: http://dx.doi.org/ 10.3233/JAD-190050.

\section{REFERENCES}

[1] World Health Organization (2017) Dementia Fact Sheet, https://www.who.int/news-room/fact-sheets/detail/demen tia, Last updated December, 2017, Accessed on 26 October, 2018.

[2] Wijngaarden E Van, Wedden H Van Der, Henning Z, Komen R (2018) Entangled in uncertainty: The experience of living with dementia from the perspective of family caregivers. PLoS One 13, 1-21.

[3] AAL Programme (2018) https://www.aal-europe.eu/, Accessed on 23 October, 2018

[4] Evans J, Brown M, Coughlan T, Lawson G, Craven M (2015) A systematic review of dementia focused assistive technology. Lecture Notes in Computer Science 9170, 3-12.

[5] Greenhalgh T, Wherton J, Sugarhood P, Hinder S, Procter R, Stones R (2013) What matters to older people with assisted living needs? A phenomenological analysis of the use and non-use of telehealth and telecare. Soc Sci Med 93, 86-94.

[6] International Organization for Standardization (2015) ISO 9241-210:2010 - Ergonomics of human-system interaction - Part 210: Human-centred design for interactive systems, https://www.iso.org/standard/52075.html,
Last updated December, 2015, Accessed on 9 November, 2018.

[7] Koskinen I, Zimmerman J, Binder T, Redstrom J, Wensveen S (2012) Design Research Through Practice. Elsevier Inc., Waltham.

[8] Sanders EB-N, Stappers PJ (2014) Probes, toolkits and prototypes: Three approaches to making in codesigning. CoDesign 10, 5-14.

[9] Steen M (2011) Tensions in human-centred design. CoDesign 7, 45-60.

[10] Hendriks N, Slegers K, Duysburgh P (2015) Codesign with people living with cognitive or sensory impairments: A case for method stories and uniqueness. CoDesign 11, 70-82.

[11] Topo P (2009) Technology Studies to Meet the Needs of People With Dementia and Their Caregivers.

[12] Span M, Hettinga M, Vernooij-Dassen M, Eefsting J, Smits C (2013) Involving people with dementia in the development of supportive IT applications: A systematic review. Ageing Res Rev 12, 535-551.

[13] Meiland F, Innes A, Mountain G, Robinson L, van der Roest H, García-Casal JA, Gove D, Thyrian JR, Evans S, Dröes R-M, Kelly F, Kurz A, Casey D, Szcześniak D, Dening T, Craven MP, Span M, Felzmann H, Tsolaki M, Franco-Martin M (2017) Technologies to support community-dwelling persons with dementia: A position paper on issues regarding development, usability, effectiveness and cost-effectiveness, deployment, and ethics. JMIR Rehabil Assist Technol 4, e1.

[14] Holthe T, Halvorsrud L, Karterud D, Hoel K-A, Lund A (2018) Usability and acceptability of technology for community-dwelling older adults with mild cognitive impairment and dementia: A systematic literature review. Clin Interv Aging 13, 863-886.

[15] Sanders EB-N, Stappers PJ (2008) Co-creation and the new landscapes of design. CoDesign 4, 5-18.

[16] Hanson E, Magnusson L, Arvidsson H, Claesson A, Keady J, Nolan M (2007) Working together with persons with early stage dementia and their family members to design a user-friendly technology-based support service. Dementia 6, 411-434.

[17] Lindsay S, Jackson D, Ladha C, Ladha K, Brittain K, Olivier P (2012) Empathy, participatory design and people with dementia. CHI' 12 Proceedings of the SIGCHI Conference on Human Factors in Computing Systems, pp. 521-530

[18] Hendriks N, Slegers K, Duysburgh P (2015) Codesign with people living with cognitive or sensory impairments: A case for method stories and uniqueness. CoDesign 11, 70-82.

[19] Bland JM, Altman DG (1986) Statistical methods for assessing agreement between two methods of clinical measurement. Lancet 327, 307-310.

[20] Pluye P, Robert E, Cargo M, Bartlett G (2011) Proposal: A mixed methods appraisal tool for systematic mixed studies reviews. Montréal McGill Univ. 1-8.

[21] Steen M (2008) The fragility of human-centred design (Doctoral dissertation). Retrieved from https://repository. tudelft.nl/

[22] Preece J, Rogers Y, Sharp H (2015) Interaction design: Beyond human-computer interaction (4th edition). John Wiley \& Sons Ltd, Chicester.

[23] De Beer R, Hol S, Keijers R, Shahid S, Al Mahmud A, Mubin O (2010) PMD: Designing a portable medicine dispenser for persons suffering from Alzheimer's disease. In The 12th International Conference on Computers Helping People with Special Needs (ICCHP 2010). Springer/Link, Berlin/Heidelberg, pp. 332-335. 
[24] Morrissey K, Wood G, Green D, Pantidi N, McCarthy J (2016) "I'm a rambler, I'm a gambler, I'm a long way from home." Proceedings of DIS '16 2016 ACM Conference on Designing Interactive Systems. Association for Computing Machinery, pp. 1008-1020.

[25] Bennett P, Hinder H, Cater K (2016) Rekindling imagination in dementia care with the resonant interface rocking chair. CHI EA '16 Proceedings of the 2016 CHI Conference: Extended Abstracts on Human Factors in Computing Systems. Association for Computing Machinery (ACM), New York, pp. 2020-2026.

[26] Wu C-L, Wu B-H, Lin Y-T, Wang P, Zhou Y (2016) Moments: A wearable device for early stage AD patients to maintain their autonomy. In CHI EA' 16 Proceedings of the 2016 CHI Conference Extended Abstracts on Human Factors in Computing Systems, pp. 74-79.

[27] Ly NT, Hurtienne J, Tscharn R, Aknine S, Serna A (2015) Towards intelligent and implicit assistance for people with dementia: Support for orientation and navigation. Proceedings of the XVI International Conference on Human Computer Interaction. New York, pp. 31:1-31:4.

[28] Schneider C, Willner V, Feichtenschlager M, Andrushevich A, Spiru L (2013) Collecting user requirements for electronic assistance for people with dementia: A case study in three countries. In eHealth 2013, Ammenwerth E, Hörbst A, Hayn D, Schreier G, eds. OCG, Vienna, pp. 107-112.

[29] Boyd H, Jones S, Harris N, Panteli N, Leake J, Jones R (2014) Development and testing of the inTouch video link for people with dementia: Design approach and practical challenges. IEEE International Conference on Bioinformatics and Biomedicine (BIBM), pp. 608-612.

[30] Donnelly M, Nugent C, McClean S, Scotney B, Mason S, Passmore P, Craig D (2010) A mobile multimedia technology to aid those with Alzheimer's disease. IEEE Multimed 17, 42-51.

[31] Huldtgren A, Mertl F, Vormann A, Geiger C (2016) Reminiscence of people with dementia mediated by a tangible multimedia book. 2nd International Conference on Information and Communication Technologies for Ageing Well and e-Health, pp. 191-201.

[32] [32] Lazar A, Edasis C, Piper AM (2017) Supporting people with dementia in digital social sharing. In Proceedings of ACM Conference on Human Factors in Computing Systems (CHI 2017), New York, pp. 2149-2162.

[33] Jordan P, Silva PA, Nunes F, Oliveira R (2013) MobileWAY - A system to reduce the feeling of temporary lonesomeness of persons with dementia and to foster inter-caregiver collaboration. 46th Hawaii International Conference on System Sciences, pp. 2474-2483.

[34] Folstein MF, Folstein SE, McHugh PR (1975) "Mini-mental state": A practical method for grading the cognitive state of patients for the clinician. J Psychiatr Res 12, 189-198.

[35] Alm N, Astell A, Ellis M, Dye R, Gowans G, Campbell J (2004) A cognitive prosthesis and communication support for people with dementia. Neuropsychol Rehabil 14, 117134.

[36] Jacova C, McGrenere J, Lee HS, Wang WW, Le Huray S, Corenblith EF, Brehmer M, Tang C, Hayden S, Beattie BL, Hsiung GY (2015) C-TOC (Cognitive Testing on Computer): Investigating the usability and validity of a novel self-administered cognitive assessment tool in aging and early dementia. Alzheimer Dis Assoc Disord 29, 213-221.

[37] van Rijn H, van Hoof J, Stappers PJ (2010) Designing leisure products for people with dementia: Developing " "the
Chitchatters"' game. Am J Alzheimers Dis Other Demen 25, 74-89.

[38] Hagethorn FN, Kröse BJA, de Greef P, Helmer ME (2008) Creating design guidelines for a navigational aid for mild demented pedestrians. In Ambient Intelligence. AmI 2008. Lecture Notes in Computer Science, vol 5355, Aarts et al., eds. Springer, Berlin, Heidelberg, pp. 276-289.

[39] Holbø K, Bøthun S, Dahl Y (2013) Safe walking technology for people with dementia: What do they want? Proceedings of the 15th International ACM SIGACCESS Conference on Computers and Accessibility, 1-8.

[40] Lee ML, Dey AK (2007) Providing good memory cues for people with episodic memory impairment. Proceedings of the 9th International ACM SIGACCESS Conference on Computers and Accessibility, pp. 131-138.

[41] Lopes P, Pino M, Carletti G, Hamidi S, Legué S, Kerhervé H, Benveniste S, Andéol G, Bonsom P, Reingewirtz S, Rigaud A (2016) Co-conception process of an innovative assistive device to track and find misplaced everyday objects for older adults with cognitive impairment: The TROUVE Project. IRBM 37, 52-57.

[42] Cohene T, Baecker R, Marziali E (2005) Designing interactive life story multimedia for a family affected by Alzheimer's disease. CHI EA '05 CHI '05 Extended Abstracts on Human Factors in Computing Systems, pp. 1300-1303.

[43] Boman I-L, Lundberg S, Starkhammar S, Nygård L (2014) Exploring the usability of a videophone mock-up for persons with dementia and their significant others. BMC Geriatr 14, 49.

[44] Kerkhof YJF, Rabiee F, Willems CG (2015) Experiences of using a memory aid to structure and support daily activities in a small-scale group accommodation for people with dementia. Dementia (London) 14, 633-649.

[45] Wang RH, Mihailidis A, Sudhama A, Begum M, Huq R (2017) Robots to assist daily activities: Views of older adults with Alzheimer's disease and their caregivers. Int Psychogeriatr 29, 67-79.

[46] Meiland F, Bourman AI, Savenstedt S, Bentvelzen S, Davies RJ, Mulvenna MD, Nugent CD, Moelaert F, Hettinga ME, Bengtsson JE, Droes RM, Bouman AIE, Sävenstedt S, Bentvelzen S, Davies RJ, Mulvenna MD, Nugent CD, Moelaert F, Hettinga ME, Bengtsson JE, Dröes R-M (2012) Usability of a new electronic assistive device for community-dwelling persons with mild dementia. Aging Ment Health 16, 584-591.

[47] Span M, Hettinga M, Groen-van de Ven L, Jukema J, Janssen R, Vernooij-Dassen M, Eefsting J, Smits C (2017) Involving people with dementia in developing an interactive web tool for shared decision-making: Experiences with a participatory design approach. Disabil Rehabil 40, 1410-1420.

[48] Meiland F, Hattink BJJ, Overmars-Marx T, de Boer ME, Jedlitschka A, Ebben PWG, Stalpers-Croeze IINW, Flick S, van der Leeuw J, Karkowski IP, Dröes RM (2014) Participation of end users in the design of assistive technology for people with mild to severe cognitive problems; the European Rosetta project. Int Psychogeriatr 26, 769-79.

[49] Span M, Smits C, Jukema J, de Ven LG van, Janssen R, Vernooij-Dassen M, Eefsting J, Hettinga M (2015) An interactive web tool for facilitating shared decision-making in dementia-care networks: A field study. Front Aging Neurosci 7, 1-12.

[50] Tobiasson H, Sundblad Y, Walldius $\AA$, Hedman A (2015) Designing for active life: Moving and being moved together with dementia patients. Int J Des 9, 47-62. 
[51] Mayer JM, Zach J (2013) Lessons learned from participatory design with and for people with dementia. Proceedings of the 15th international conference on Human-computer interaction with mobile devices and services (Germany, Munich, 2013), pp. 540-545.

[52] Dethlefs N, Milders M, Cuayáhuitl H, Al-Salkini T, Douglas L (2017) A natural language-based presentation of cognitive stimulation to people with dementia in assistive technology: A pilot study. Inform Health Soc Care 42, 349-360.

[53] Boman I-L, Nygård L, Rosenberg L (2014) Users' and professionals' contributions in the process of designing an easy-to-use videophone for people with dementia. Disabil Rehabil Assist Technol 3107, 164-172.

[54] Hattink B, Meiland F, Overmars-Marx T, de Boer M, Ebben PWG, van Blanken M, Verhaeghe S, Stalpers-Croeze I, Jedlitschka A, Flick SE, v/d Leeuw J, Karkowski I, Dröes RM (2016) The electronic, personalizable Rosetta system for dementia care: Exploring the user-friendliness, usefulness and impact. Disabil Rehabil Assist Technol 11, 61-71.

[55] Suijkerbuijk S, Brankaert R, De Kort YAW, Snaphaan LJAE, Den Ouden E (2015) Seeing the first-person perspective in dementia: A qualitative personal evaluation game to evaluate assistive technology for people affected by dementia in the home context. Interact Comput 27, 47-59.

[56] Gibson A, McCauley C, Mulvenna M, Ryan A, Laird L, Curran K, Bunting B, Ferry F, Bond R (2016) Assessing usability testing for people living with dementia. Proceedings of the 4th Workshop on ICTs for improving Patients Rehabilitation Research Techniques, pp. 25-31.

[57] Peeters MMM, Harbers M, Neerincx MA (2016) Designing a personal music assistant that enhances the social, cognitive, and affective experiences of people with dementia. Comput Hum Behav 63, 727-737.

[58] Olsson A, Engström M, Lampic C, Skovdahl K (2013) A passive positioning alarm used by persons with dementia and their spouses - a qualitative intervention study. $B M C$ Geriatr 13, 11.

[59] Orpwood R, Gibbs C, Adlam T, Faulkner R, Meegahawatte D (2005) The design of smart homes for people with dementia - User-interface aspects. Univers Access Inf Soc 4, 156-164.

[60] Lazar A, Edasis C, Piper AM (2017) A critical lens on dementia and design in HCI. In Proceedings of the 2017 CHI Conference on Human Factors in Computing Systems ACM, New York, pp. 2175-2188.

[61] Nunes F, Verdezoto N, Fitzpatrick G, Kyng M, Grönvall E, Storni C (2015) Self-care technologies in HCI: Trends, tensions, and opportunities. ACM Trans Comput Interact 22, $33: 1-33: 45$.

[62] Blandford A, Gibbs J, Newhouse N, Perski O, Singh A, Murray E (2018) Seven lessons for interdisciplinary research on interactive digital health interventions. Digit Health 4, 2055207618770325.

[63] Brankaert R, Ouden E Den, Brombacher A (2015) Innovate dementia: The development of a living lab protocol to evaluate interventions in context. ISM-Info 17, 40-52.

[64] Astell A, Alm N, Gowans G, Ellis M, Dye R, Vaughan P (2009) Involving older people with dementia and their carers in designing computer based support systems: Some methodological considerations. Univers Access Inf Soc 8, 49-58.

[65] Wolters MK, Kelly F, Kilgour J (2016) Designing a spoken dialogue interface to an intelligent cognitive assistant for people with dementia. Health Informatics J 22, 854-866.
[66] Van der Roest HG, Wenborn J, Pastink C, Dröes RM, Orrell M (2017) Assistive technology for memory support in dementia. Cochrane Database Syst Rev 26, CD009627.

[67] Branco RM, Quental J, Ribeiro Ó (2017) Personalised participation: An approach to involve people with dementia and their families in a participatory design project. CoDesign $\mathbf{1 3}$, 127-143.

[68] Morrissey K, McCarthy J, Pantidi N (2017) The value of experience-centred design approaches in dementia research contexts. Proceedings of the 2017 CHI Conference on Human Factors in Computing Systems, pp. 1326-1338.

[69] Brooker D, Latham I (2015) Person-centred dementia care: Making services better with the VIPS framework. Jessica Kingsley Publishers, London.

[70] Karlsson E, Axelsson K, Zingmark K, Savenstedt S (2011) The challenge of coming to terms with the use of a new digital assistive device: A case study of two persons with mild dementia. Open Nurs J 5, 102-110.

[71] Pakrasi S, Burmeister OK, Coppola JF, McCallum TJ, Loeb $G$ (2015) Ethical telehealth design for users with dementia. Gerontechnology 13, 383-387.

[72] McCabe L, Innes A (2013) Supporting safe walking for people with dementia: User participation in the development of new technology. Gerontechnology 12, 4-15.

[73] Wang RH, Gorski SM, Holliday PJ, Fernie GR (2011) Evaluation of a contact sensor skirt for an anti-collision power wheelchair for older adult nursing home residents with dementia: Safety and mobility. Assist Technol 23, 117-134.

[74] Hattink B, Droes R-M, Sikkes S, Oostra E, Lemstra AW (2016) Evaluation of the Digital Alzheimer Center: Testing usability and usefulness of an online portal for patients with dementia and their carers. JMIR Res Protoc 5, e144.

[75] Begum M, Wang R, Huq R, Mihailidis A (2013) Performance of daily activities by older adults with dementia: The role of an assistive robot. In 2013 IEEE 13th International Conference on Rehabilitation Robotics (ICORR), pp. 1-8.

[76] Nugent C, O'Neill S, Donnelly M, Parente G, Beattie M, McClean S, Scotney B, Mason S, Craig D (2011) Evaluation of video reminding technology for persons with dementia. In Toward Useful Services for Elderly and People with Disabilities. ICOST 2011. Lecture Notes in Computer Science, vol 6719. Abdulrazak B, Giroux S, Bouchard B, Pigot H, Mokhtari M, eds. Springer, Berlin, Heidelberg, pp. 153-160.

[77] Alm N, Dye R, Astell A, Ellis M, Gowans G, Campbell J (2005) Making software accessible to people with severe memory deficits. Accessible Design'05 Proceedings of the 2005 international conference on Accessible Design in the Digital World, pp. 16.

[78] Morán AL, Meza Kubo V (2009) Towards a tele-assistance service for the cognitive stimulation of elders with cognitive decline. 2009 International Conference on eHealth, Telemedicine, and Social Medicine, pp. 160-165.

[79] Wan L, Müller C, Randall D, Wulf V (2016) Design of A GPS monitoring system for dementia care and its challenges in academia-industry project. ACM Trans Comput Interact 23, 1-36.

[80] König A, Malhotra A, Hoey J, Francis LE (2016) Designing personalized prompts for a virtual assistant to support elderly care home residents. Proceedings of the 10th EAI International Conference on Pervasive Computing Technologies Healthcare, pp. 278-282.

[81] Nugent C, Davies R, Donnelly M, Hallberg J, Hariz M, Craig D, Meiland F, Moelaert F, Bengtsson J, Savenstedt S, Mulvenna M, Dröes R (2008) The development of personalised cognitive prosthetics. In 30th Annual International IEEE 
EMBS Conference Vancouver, British Columbia, Canada, pp. 787-790.

[82] Nugent C, Moelaert F, Davies R, Donnelly M, Savenstedt S, Meiland F, Droes RM, Hettinga M, Craig D, Mulvenna M, Bengstsson JE (2008) Evaluation of mobile and home based cognitive prosthetics. In Smart Homes and Health Telematics. ICOST 2008. Lecture Notes in Computer Science, vol 5120, Helal S, Mitra S, Wong J, Chang CK, Mokhtari M, eds. Springer, Berlin, Heidelberg, pp. 18-25.

[83] Ly NT, Tscharn R, Preßler J, Huber S, Aknine S, Serna A, Hurtienne J (2016) Smart lighting in dementia care facility. Proceedings of the 2016 ACM International Joint Conference on Pervasive and Ubiquitous Computing: Adjunct, pp. 1636-1639. 